\title{
ADAPTED METRICS FOR SINGULAR HYPERBOLIC FLOWS
}

\author{
VITOR ARAUJO, VINICIUS COELHO, AND LUCIANA SALGADO
}

\begin{abstract}
Singular and sectional-hyperbolic sets are the objects of the extension of the classical Smale Hyperbolic Theory to flows having invariant sets with singularities accumulated by regular orbits within the set. It is by now well-known that (partially) hyperbolic sets admit adapted metrics.

We show the existence of singular-adapted metrics for any singular-hyperbolic set with respect to a $C^{1}$ vector field on finite dimensional compact manifolds. Moreover, we obtain sectional-adapted metrics for certain open classes of sectional-hyperbolic sets and also for any hyperbolic set.
\end{abstract}

\section{Contents}

1. Introduction 2

2. Preliminary definitions and results 3

2.1. Statements of main results 4

2.2. Applications and Conjectures 5

2.3. Organization of the text 6

Acknowledgements

3. Auxiliary results 7

3.1. Linear multiplicative cocycles over flows 7

3.2. Exterior powers 8

3.3. Dominated/partial/sectional-hyperbolic splittings and exterior powers 10

4. Proofs of main results 11

4.1. Existence of singular adapted metric 11

4.2. The uniformly hyperbolic setting 18

$\begin{array}{ll}\text { 4.3. The open class of sectional-hyperbolic sets } & 19\end{array}$

References 23

Date: October 21, 2020.

2000 Mathematics5 Subject Classification. Primary: 37D30; Secondary: 37D25;58B20.

Key words and phrases. adapted metrics, singular hyperbolicity.

V.A. is partially supported by CNPq-Brazil (grant 301392/2015-3) and FAPESB-Brazil (grand PIE0034/2016). L.S. is partially supported Fapesb-JCB0053/2013, PRODOC-UFBA/2014 and CNPq. V.C. is supported by CAPES. 


\section{INTRODUCTION}

Let $M$ be a connected compact finite $m$-dimensional manifold, $m \geq 3$, with or without boundary. We consider a vector field $X$, such that $X$ is inwardly transverse to the boundary $\partial M$, if $\partial M \neq \emptyset$. The flow generated by $X$ is denoted by $X_{t}$.

A hyperbolic set for a flow $X_{t}$ on a finite dimensional Riemannian manifold $M$ is a compact invariant set $\Gamma$ with a continuous splitting of the tangent bundle, $T_{\Gamma} M=E^{s} \oplus$ $E^{X} \oplus E^{u}$, where $E^{X}$ is the direction of the vector field, for which the subbundles are invariant under the derivative $D X_{t}$ of the flow $X_{t}$

$$
D X_{t} \cdot E_{x}^{*}=E_{X_{t}(x)}^{*}, \quad x \in \Gamma, \quad t \in \mathbb{R}, \quad *=s, X, u ;
$$

and $E^{s}$ is uniformly $(K, \lambda)$-contracted by $D X_{t}$ and $E^{u}$ is likewise $(K, \lambda)$-expanded: there are $K, \lambda>0$ so that

$$
\left\|\left.D X_{t}\right|_{E_{x}^{s}}\right\| \leq K e^{-\lambda t}, \quad\left\|\left(\left.D X_{t}\right|_{E_{x}^{u}}\right)^{-1}\right\| \leq K e^{-\lambda t}, \quad x \in \Gamma, \quad t>0 .
$$

Very strong properties can be deduced from the existence of such hyperbolic structure; see for instance $[8,9,20,12,17]$.

An important feature of a hyperbolic structure is that it does not depend on the metric on the ambient manifold; see [11]. We recall that a metric is said to be adapted to the hyperbolic structure if we can take $K=1$ in equation (1.2).

Weaker notions of hyperbolicity (e.g. dominated splitting, partial hyperbolicity, volume hyperbolicity, sectional-hyperbolicity, singular-hyperbolicity) have been developed to encompass larger classes of systems beyond the uniformly hyperbolic ones; see [7] and specifically [23, 2, 5] for singular hyperbolicity and Lorenz-like attractors.

In the same work [11], Hirsch, Pugh and Shub asked about adapted metrics for dominated splittings. This was given a positive answer by Gourmelon [10] in 2007, where adapted metrics are obtained for dominated splittings of both diffeomorphisms and flows, and also for partially hyperbolic splittings.

Proving the existence of some hyperbolic structure is, in general, a non-trivial matter, even in its weaker forms. In [13], Lewowicz proved that a diffeomorphism on a compact riemannian manifold is Anosov if and only if its derivative admits a nondegenerate Lyapunov quadratic function. An example of application of the adapted metric from [10] is contained in [3] where, following the spirit of Lewowicz's result, the authors construct quadratic forms which characterize partially hyperbolic and singular-hyperbolic structures on a trapping region for flows.

In [4], the existence of an adapted metric for singular-hyperbolic splittings was proved for three-dimensional vector fields through the use of quadratic forms. In [19, Theorem B], the existence of adapted metrics for any singular hyperbolic splitting having a onedimensional uniformly contracting bundle was obtained, extending the result from [4] for any codimension-one singular-hyperbolic set. This was done under the point of view of J-algebras of Potapov (see e.g. [25]) and through the use of quadratic forms.

Here, we extend this result for any singular-hyperbolic set and also for open classes of sectional-hyperbolic sets, using only multilinear algebra and the dynamics of the tangent map to the flow. 


\section{Preliminary Definitions And Results}

We now present preliminary definitions and results. Recall that a trapping region $U$ for a flow $X_{t}$ is an open subset of the manifold $M$ which satisfies: $X_{t}(U)$ is contained in $U$ for all $t>0$, and there exists $T>0$ such that $\overline{X_{t}(U)}$ is contained in the interior of $U$ for all $t>T$. We define $\Gamma(U)=\Gamma_{X}(U):=\cap_{t>0} \overline{X_{t}(U)}$ to be the maximal positive invariant subset in the trapping region $U$.

A singularity for the vector field $X$ is a point $\sigma \in M$ such that $X(\sigma)=\overrightarrow{0}$ or, equivalently, $X_{t}(\sigma)=\sigma$ for all $t \in \mathbb{R}$. The set formed by singularities is the singular set of $X$ denoted $\operatorname{Sing}(X)$. We say that a singularity is hyperbolic if the eigenvalues of the derivative $D X(\sigma)$ of the vector field at the singularity $\sigma$ have nonzero real part.

Definition 1. A dominated splitting over a compact invariant set $\Lambda$ of $X$ is a continuous $D X_{t}$-invariant splitting $T_{\Lambda} M=E \oplus F$ with $E_{x} \neq\{0\}, F_{x} \neq\{0\}$ for every $x \in \Lambda$ and such that there are positive constants $K, \lambda$ satisfying

$$
\left\|\left.D X_{t}\right|_{E_{x}}\right\| \cdot\left\|\left.D X_{-t}\right|_{F_{X_{t}(x)}}\right\|<K e^{-\lambda t} \text {, for all } x \in \Lambda \text {, and all } t>0 .
$$

A compact invariant set $\Lambda$ is said to be partially hyperbolic if it exhibits a dominated splitting $T_{\Lambda} M=E \oplus F$ such that subbundle $E$ is uniformly contracted, i.e., there exists $C>0$ and $\lambda>0$ such that $\left\|\left.D X_{t}\right|_{E_{x}}\right\| \leq C e^{-\lambda t}$ for $t \geq 0$. In this case $F$ is the central subbundle of $\Lambda$. Or else, we may replace uniform contraction along $E$ by uniform expansion along $F$, i.e., the right hand side condition in (1.2).

The following is an extension of the notion of 2-sectional expansion proposed in [14, Definition 2.7]. We say that a $D X_{t}$-invariant subbundle $F \subset T_{\Lambda} M$ is p-sectionally expanding if $\operatorname{dim} F_{x} \geq p$ is constant for $x \in \Lambda$ and there are positive constants $C, \lambda$ such that for every $x \in \Lambda$ and every $p$-dimensional linear subspace $L_{x} \subset F_{x}$ one has

$$
\left|\operatorname{det}\left(\left.D X_{t}\right|_{L_{x}}\right)\right|>C e^{\lambda t} \text {, for all } t>0 \text {. }
$$

Definition 2. [18, Definition 3] A p-sectional-hyperbolic set is a partially hyperbolic set whose central subbundle is $p$-sectionally expanding.

This is a particular case of the so called singular-hyperbolicity whose definition we recall now. A $D X_{t}$-invariant subbundle $F \subset T_{\Lambda} M$ is said to be volume expanding if we replace (2.2) by

$$
\left|\operatorname{det}\left(\left.D X_{t}\right|_{F_{x}}\right)\right|>C e^{\lambda t} \text {, for all } t>0 \text {. }
$$

Definition 3. [15, Definition 1] A singular-hyperbolic set is a partially hyperbolic set whose central subbundle is volume expanding.

Clearly, in the three-dimensional case, these notions are equivalent. This is a feature of the Lorenz attractor as proved in [21] and this notion extends the uniform hyperbolicity for singular flows, because sectional-hyperbolic sets without singularities are hyperbolic; see $[16,2]$. 
2.1. Statements of main results. We recall the definition of adapted metrics in the singular-hyperbolic setting, which extends the notion of adapted metric for dominated and partially hyperbolic splittings; see e.g. [10].

Definition 4. We say a Riemannian metric $\langle\cdot, \cdot\rangle$ is adapted to a partially hyperbolic splitting if it induces a norm $|\cdot|$ admitting a constant $\lambda>0$ simultaneously satisfying

$$
\left|D X_{t}\right|_{E_{x}}|\cdot|\left(\left.D X_{t}\right|_{F_{x}}\right)^{-1} \mid \leq e^{-\lambda t} ; \quad \text { and } \quad\left|D X_{t}\right|_{E_{x}} \mid \leq e^{-\lambda t}
$$

for all $x \in \Gamma$ and $t>0$. Additionally, we say that $\langle\cdot, \cdot\rangle$ is

(1) adapted to a singular-hyperbolic splitting if $|\cdot|$ also satisfies

$$
\left|\operatorname{det}\left(\left.D X_{t}\right|_{F_{x}}\right)\right| \geq e^{\lambda t}
$$

for all $x \in \Gamma$ and $t>0$. For simplicity we say that $\langle\cdot, \cdot\rangle$ is a singular-adapted metric.

(2) adapted to a sectional-hyperbolic splitting if the induced norm also satisfies

$$
\left|\operatorname{det}\left(D X_{t} \mid L_{x}\right)\right| \geq e^{\lambda t}
$$

for all $x \in \Gamma, t>0$ and 2-subspace $L_{x} \subset F_{x}$. For simplicity we say that $\langle\cdot, \cdot\rangle$ is a sectional-adapted metric.

Consider a partially hyperbolic splitting $T_{\Gamma} M=E \oplus F$ where $E$ is uniformly contracted and $F$ is volume expanding. We show that for $C^{1}$ flows having a singular-hyperbolic set $\Gamma$ there exists a metric adapted to the partial hyperbolicity and the area expansion, as follows.

Theorem A. Let $\Gamma$ be a singular-hyperbolic set for a $C^{1}$ vector field. Then $\Gamma$ admits a singular adapted metric.

In the particular case of a uniformly hyperbolic set $\Gamma$ for a flow, with splitting $T_{\Gamma} M=$ $E^{s} \oplus E^{X} \oplus E^{u}=E \oplus F$ with $E=E^{s}$ and $F=E^{X} \oplus E^{u}$ is sectional-hyperbolic and admits an adapted metric.

Theorem B. Let $\Gamma$ be a uniformly hyperbolic set for a $C^{1}$ vector field. Then $\Gamma$ admits a sectional-adapted metric.

Refining the arguments we obtain the following open class of sectional-hyperbolic attracting sets admitting sectional-adapted metrics. We say that a vector subbundle with an invariant splitting $E^{c} \oplus E^{u}$ over a compact invariant subset $\Gamma$ of a $C^{1}$ vector field is strongly partially hyperbolic if there are $K>0, \mu>\lambda>0$ so that, for each $t>0$ and $x \in \Gamma$

$$
\left\|D X_{t} \mid E_{x}^{c}\right\| \leq K e^{\lambda t} \text { and }\left\|D X_{-t} \mid E_{X_{t} x}^{u}\right\|<K e^{-\mu t} .
$$

Note that this is clearly stronger than the domination condition.

Theorem C. Let $\Gamma$ be a partially hyperbolic set for a $C^{1}$ vector field whose splitting $T_{\Gamma} M=$ $E^{s} \oplus E^{c} \oplus E^{u}$ is dominated with subbundles of constant dimension and such that $E^{s}$ is uniformly contracted; $E^{c}$ is 2-dimensional and area expanding; $E^{u}$ is uniformly expanded; and $E^{c} \oplus E^{u}$ is strongly partially hyperbolic. Then $\Gamma$ admits a sectional-adapted metric. 


\subsection{Applications and Conjectures.}

Example 1. In [22] Turaev and Shilnikov construct a wild attractor $\Gamma$ (see Figure 1) of a vector field having a singular hyperbolic splitting $T_{\Gamma} M=E \oplus F$ where $\operatorname{dim} F=3$ and $\operatorname{dim} E=n-3$ for any $n \geq 4$. Moreover, the bundle $F$ is not sectionally expanding; see [22, p 296, formula (13)]. These attractors are also robust: these properties persist for all $C^{1}$ small perturbations of the given vector field.

Hence Theorem A ensures that the wild attractors constructed in [22] admit a singularhyperbolic metric.

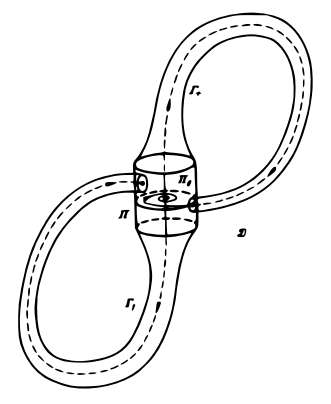

Figure 1. Example of Turaev-Shil'nikov's wild strange attractor.

For the next application, we recall that the Riemannian manifold $M$ is naturally endowed with a volume form $m$ called Lebesgue measure.

Corollary 2.1. Let $X$ be a $C^{1}$ volume preserving flow on $M$. Then every partially hyperbolic set is singular-hyperbolic and thus admits a singular-adapted metric.

Proof. It is enough to explain that partial hyperbolicity of a compact invariant subset $\Gamma$ for a conservative vector field $X$ implies singular-hyperbolicity, as follows.

Let $T_{\Gamma} M=E^{s} \oplus F$ be the singular hyperbolic splitting over $\Gamma$. Domination and compactness imply that the minimum angle

$$
\varangle\left(E^{s}, F\right)=\inf \left\{\arccos \langle u, v\rangle: u \in E_{x}^{s}, v \in F_{x},\|u\|=\|v\|=1, x \in \Gamma\right\}
$$

between the bundles is away from zero; see [7, Appendix B]. Recall that $E^{s}$ is uniformly contracted. Hence, $\left|\operatorname{det} D X_{t}\right| \equiv 1$ since $X$ is conservative and for all $x \in \Gamma, t>0$

$$
1=\left|\operatorname{det} D X_{t}(x)\right|=\left|\operatorname{det} D X_{t}\right| E_{x}^{s}|\cdot| \operatorname{det} D X_{t}\left|F_{x}\right| \cdot \sin \varangle\left(E_{x}^{s}, F_{x}\right)
$$

so that

$$
\left|\operatorname{det} D X_{t}\right| F_{x} \mid=\left(\left|\operatorname{det} D X_{t}\right| E_{x}^{s} \mid \cdot \sin \varangle\left(E_{x}^{s}, F_{x}\right)\right)^{-1} \geq\left(K e^{-\mu t} \sin \varangle\left(E^{s}, F\right)\right)^{-1}
$$

is uniformly expanding.

Our methods do not provide that the singular-adapted metric is still preserved by the flow.

Conjecture 1. Given a singular hyperbolic set for a conservative $C^{1}$ vector field, there exists a singular-adapted metric whose induced volume form is still preserved by the flow. 
Example 2. An open class of examples in the setting of Theorem $\mathrm{C}$ can be provided by modifying the classical Lorenz equations as follows.

$$
\dot{X}=a(Y-X) ; \quad \dot{Y}=r X-Y-X Z ; \quad \dot{Z}=X Y-b Z ; \quad \dot{W}=r W ;
$$

where $(X, Y, Z) \in \mathbb{R}^{3}, W \in \mathbb{R}^{k}$ for some fixed $k>1$ and also $a=10, b=8 / 3$ and $r=28$.

Let $G$ be the vector field on $\mathbb{R}^{3}$ provided by the first three equations above on $(X, Y, Z)$ and $H$ the vector field on $\mathbb{R}^{k}$ given by the last equation on $W$. Then the vector field on $\mathbb{R}^{k+3}$ given by the full set of equations can be written as the direct product $J=G \times H$. We write $\phi_{t}$ for the flow of $G$ and $\psi_{t}$ for the flow of $J$.

Let $U$ be the open trapping region of the Lorenz attractor $\Lambda$ in $\mathbb{R}^{3}$, so that $\Lambda=\cap_{t \in \mathbb{R}} \phi_{t}(U)$. Then the compact invariant subset $\tilde{\Lambda}=\Lambda \times\left\{0^{k}\right\}=\cap_{t \in \mathbb{R}} \psi_{t}(U \times V)$ of the vector field $J$, where $V$ is any open neighborhood of $0^{k}$ in $\mathbb{R}^{k}$, admits a sectional-hyperbolic splitting $E^{s} \oplus F$ with:

- $E^{s}$ corresponding to the one-dimensional stable (uniformly contracting) subbundle of $T_{\Lambda} \mathbb{R}^{3} ;$ and

- $F$ admits a strongly partially hyperbolic splitting $F=E^{c} \oplus E^{u}$, where

- $E^{c}$ corresponds to the two-dimensional sectional-expanding subbundle of $T_{\Lambda} \mathbb{R}^{3}$; and

- $E^{u}$ is the uniformly expanded $k$-dimensional subbundle $0^{3} \times \mathbb{R}^{k}$.

For the properties of the subbundles $E^{s}, E^{c}$ the reader may consult [2] and references therein.

This example is clearly robust on the $C^{1}$ topology of vector fields: there exists a neighborhood $\mathcal{U}$ of $W$ so that each $Y \in \mathcal{U}$ admits a maximal invariant subset $\Lambda_{Y}=\cap_{t \in \mathbb{R}} Y_{t}(U \times V)$ exhibiting a splitting $T_{\Lambda_{Y}}=E_{\Lambda_{Y}} \oplus F_{\Lambda_{Y}}$ with the same properties as above.

Hence, Theorem $\mathrm{C}$ ensures that the splitting of $T_{\Lambda_{Y}}$ admits an adapted sectionalhyperbolic metric for each $Y \in \mathcal{U}$.

It is now natural to conjecture that the conclusion of Theorem $\mathrm{C}$ holds in a much more general setting.

Conjecture 2. Given a $p$-sectional-hyperbolic set $\Gamma$ for a $C^{1}$ vector field $X$, there exists a metric such that for some constant $\mu>0$ and all $t>0$

- $\left|D X_{t}\right|_{E_{x}} \mid \leq e^{-\mu t}$;

- $\left|D X_{t}\right|_{E_{x}}|\cdot|\left(\left.D X_{t}\right|_{F_{x}}\right)^{-1} \mid \leq e^{-\mu t}$; and

- $\left|\wedge^{p} D X_{t}(x)\right|_{L_{x}} \mid>e^{\mu t}$ for every $p$-dimensional linear subspace $L_{x} \subset F_{x}$.

2.3. Organization of the text. In the present section we provided preliminary definitions in order to present the statements of the main results together with some applications and conjectures. In Section 3 we state some auxiliary results, definitions and prove some useful properties of exterior products. In Section 4 we give the proofs of our theorems, divided into three subsections 4.1, 4.2 and 4.3, one for each of the Main Theorems A, B and C, respectively. 
Acknowledgements. This work is part of the PhD. Thesis of V. Coelho developed at the Mathematics Department of the Federal University of Bahia (UFBA) under the supervision of L. Salgado. The authors would like to thank the facilities provided by the Mathematics Institute for the PhD. Program and the partial financial support from several federal and state agencies to the Faculty and Students of this Program.

\section{Auxiliary Results}

3.1. Linear multiplicative cocycles over flows. Let $A: G \times \mathbb{R} \rightarrow G$ be a smooth map given by a collection of linear bijections

$$
A_{t}(x): G_{x} \rightarrow G_{X_{t}(x)}, \quad x \in \Gamma, t \in \mathbb{R}
$$

where $\Gamma$ is the base space of the finite dimensional vector bundle $G$, satisfying the cocycle property

$$
A_{0}(x)=I d, \quad A_{t+s}(x)=A_{t}\left(X_{s}(x)\right) \circ A_{s}(x), \quad x \in \Gamma, t, s \in \mathbb{R},
$$

with $\left\{X_{t}\right\}_{t \in \mathbb{R}}$ a complete smooth flow over $M \supset \Gamma$. We note that for each fixed $t>0$ the map $A_{t}: G \rightarrow G, v_{x} \in G_{x} \mapsto A_{t}(x) \cdot v_{x} \in G_{X_{t}(x)}$ is an automorphism of the vector bundle $G$.

The natural example of a linear multiplicative cocycle over a smooth flow $X_{t}$ on a manifold is the derivative cocycle $A_{t}(x)=D X_{t}(x)$ on the tangent bundle $G=T M$ of a finite dimensional compact manifold $M$. Another example is given by the exterior power $A_{t}(x)=\wedge^{k} D X_{t}$ of $D X_{t}$ acting on $\wedge^{k} T M$, the family of all $k$-vectors on the tangent spaces of $M$, for some fixed $1 \leq k \leq \operatorname{dim} G$.

It is well-known that the exterior power of an inner product space has a naturally induced inner product and thus a norm. Thus $G=\wedge^{k} T M$ has an induced norm from the Riemannian metric of $M$. For more details see e.g. [6].

In what follows we assume that the vector bundle $G$ has a smoothly defined inner product in each fiber $G_{x}$ which induces a corresponding norm $\|\cdot\|_{x}, x \in \Gamma$.

Definition 5. A continuous splitting $G=E \oplus F$ of the vector bundle $G$ into a pair of subbundles is dominated (with respect to the automorphism $A$ over $\Gamma$ ) if

- the splitting is invariant: $A_{t}(x) \cdot E_{x}=E_{X_{t}(x)}$ and $A_{t}(x) \cdot F_{x}=F_{X_{t}(x)}$ for all $x \in \Gamma$ and $t \in \mathbb{R}$; and

- there are positive constants $K, \lambda$ satisfying

$$
\left\|\left.A_{t}\right|_{E_{x}}\right\| \cdot\left\|\left.A_{-t}\right|_{F_{X_{t}(x)}}\right\|<K e^{-\lambda t} \text {, for all } x \in \Gamma \text {, and all } t>0 \text {. }
$$

We say that the splitting $G=E \oplus F$ is partially hyperbolic if it is dominated and the subbundle $E$ is uniformly contracted: $\left\|A_{t} \mid E_{x}\right\| \leq C e^{-\mu t}$ for all $t>0$ and suitable constants $C, \mu>0$. 
3.2. Exterior powers. Let $V$ be a vector space of dimension $N$. The $k$ th exterior power of $V$, denoted $\wedge^{k}(V)$, is the vector space spanned by alternating (exterior) products of the form

$$
x_{1} \wedge x_{2} \wedge \cdots \wedge x_{k}, \quad x_{i} \in V, i=1,2, \ldots, k .
$$

Every $w \in \wedge^{k}(V)$ is said to be a $k$-vector. If $w$ can be expressed as an exterior product of $k$ elements of $V$, then $w$ is decomposable. Decomposable $k$-vectors $\operatorname{span} \wedge^{k}(V)$ but not every element of $\wedge^{k}(V)$ is decomposable. ${ }^{1}$ Since the exterior product is alternating, then $\wedge^{N+r} V$ is the trivial vector space for each integer $r>0$.

Remark 3.1. (1) The dimension of space $\wedge^{r} V$ is $\operatorname{dim} \wedge^{r} V=\left(\begin{array}{l}N \\ r\end{array}\right)$ for each $1 \leq r \leq N$. If $\left\{e_{1}, \cdots, e_{N}\right\}$ is a basis of $V$, so the set $\left\{e_{k_{1}} \wedge \cdots \wedge e_{k_{r}}: 1 \leq k_{1}<\cdots<k_{r} \leq N\right\}$ is a basis in $\wedge^{r} V$ with $\left(\begin{array}{l}N \\ r\end{array}\right)$ elements.

(2) Each $r$-vector $v_{1} \wedge \cdots \wedge v_{r}$ can be represented by $v_{1} \wedge \tilde{v}_{2} \wedge \cdots \wedge \tilde{v}_{r}$, where $v_{1}, \tilde{v_{2}} \ldots, \tilde{v}_{r}$ are mutually orthogonal and each $\tilde{v}_{i}$ belongs to the span of $\left\{v_{1}, \ldots, v_{i}\right\}$, for each $i=2, \ldots, r$ (just apply the Gram-Schmidt ortogonalization procedure).

(3) If $V$ admits an inner product $\langle\cdot, \cdot\rangle$, then the bilinear extension of

$$
\left\langle u_{1} \wedge \cdots \wedge u_{r}, v_{1} \wedge \cdots \wedge v_{r}\right\rangle:=\operatorname{det}\left(\left\langle u_{i}, v_{j}\right\rangle\right)_{r \times r}
$$

defines an inner product in $\wedge^{r} V$. In particular, $\left\|u_{1} \wedge \cdots \wedge u_{r}\right\|=\sqrt{\operatorname{det}\left(\left\langle u_{i}, u_{j}\right\rangle\right)_{r \times r}}$ is a norm on $\wedge^{r} V$ and is also the volume of the $r$-dimensional parallelepiped $H$ spanned by $u_{1}, \cdots, u_{r}: \operatorname{vol}\left(u_{1}, \cdots, u_{r}\right)=\operatorname{vol}(H)=\operatorname{det}(H)=\left|\operatorname{det}\left(u_{1}, \cdots, u_{r}\right)\right|$.

(4) If $A: V \rightarrow V$ is a linear operator then the linear extension of $\wedge^{r} A\left(u_{1} \wedge \cdots \wedge u_{r}\right)=$ $A\left(u_{1}\right) \wedge \cdots \wedge A\left(u_{r}\right)$ defines a linear operator $\wedge^{r} A$ on $\wedge^{r} V$.

(5) Let $A: V \rightarrow V$ be a linear operator, $\wedge^{r} A: \wedge^{r} V \rightarrow \wedge^{r} V$ and $G$ a subspace of $V$ spanned by $v_{1}, \cdots, v_{s} \in V$. Define $H:=\left.A\right|_{G}$. Then $H(G)$ is spanned by $A\left(v_{1}\right), \cdots, A\left(v_{s}\right)$ and $|\operatorname{det} A|_{G} \mid=\operatorname{vol}\left(\left.A\right|_{G}\right)=\operatorname{vol}(H)=\operatorname{vol}\left(A\left(v_{1}\right), \cdots, A\left(v_{s}\right)\right)=$ $\left\|A\left(v_{1}\right) \wedge \cdots \wedge A\left(v_{s}\right)\right\|=\left\|\wedge^{s} A\left(v_{1} \wedge \cdots \wedge v_{s}\right)\right\|$.

3.2.1. Exterior power of linear multiplicative cocycles. The algebraic construction of exterior power of a vector space can be applied to any given fiber of a vector bundle and thus obtain the exterior power of the vector bundle, whose fibers are the corresponding exterior powers of the original fibers.

It is natural to consider the linear multiplicative cocyle $\wedge^{k} D X_{t}$ on $\wedge{ }^{k} T_{U} M$ over the flow $X_{t}$ of $X$ on $U$, that is, for any $k$ choice, $u_{1}, u_{2}, \cdots, u_{k}$ of vectors in $T_{x} M, x \in U$ and $t \in \mathbb{R}$ such that $X_{t}(x) \in U$ we set

$$
\left(\wedge^{k} D X_{t}\right) \cdot\left(u_{1} \wedge u_{2} \wedge \cdots \wedge u_{k}\right)=\left(D X_{t} \cdot u_{1}\right) \wedge\left(D X_{t} \cdot u_{2}\right) \wedge \cdots \wedge\left(D X_{t} \cdot u_{k}\right)
$$

see e.g. [6, Chapter 3, Section 2.3] or [24] for more details and standard results on exterior algebra and exterior products of linear operators.

\footnotetext{
${ }^{1}$ For example, in $\mathbb{R}^{4}$ the following 2-vector (a symplectic form) is not decomposable: $e_{1} \wedge e_{2}+e_{3} \wedge e_{4}$.
} 
Remark 3.2. In particular, if $D X_{t}\left(u_{i}\right)=v_{i}(t)=v_{i}$, where $G$ is a subspace of $T_{x} M$ spanned by $u_{1}, \cdots, u_{r} \in T_{x} M$, then $H=D X_{t}(G)$ is spanned by $v_{1}, \cdots, v_{r}$. Thus

$$
\begin{aligned}
\left|\operatorname{det}\left(\left.D X_{t}\right|_{G}\right)\right| & =\operatorname{vol}\left(D X_{t}\left(u_{1}\right), \cdots, D X_{t}\left(u_{r}\right)\right) \\
& =\left\|D X_{t}\left(u_{1}\right) \wedge \cdots \wedge D X_{t}\left(u_{r}\right)\right\|=\left\|\wedge^{r} D X_{t}\left(u_{1} \wedge \cdots \wedge u_{r}\right)\right\| .
\end{aligned}
$$

3.2.2. Conditions for sectional expansion. Let $\Gamma$ be a compact invariant set of $X$ such that it exhibits a continuous $D X_{t}$-invariant splitting $E \oplus F$ on a subbundle of $T_{\Gamma} M$, with $E_{x} \neq\{0\}, F_{x} \neq\{0\}$ for every $x \in \Gamma$.

If $\left\{e_{1}, \ldots, e_{\ell}\right\}$ is a basis for $E$ and $\left\{f_{1}, \ldots, f_{m}\right\}$ is a basis for $F$ (where the $e_{i}$ amd $f_{k}$ are vector fields over $\Gamma$ ), then $\widetilde{F}=\wedge^{k} F$ generated by $\left\{f_{i_{1}} \wedge \cdots \wedge f_{i_{k}}\right\}_{1 \leq i_{1}<\cdots<i_{k} \leq m}$ is a subbundle of $\wedge^{k} T_{\Gamma} M$ which naturally is $\wedge^{k} D X_{t}$-invariant by construction.

In addition, $\tilde{E}=E \wedge\left(\wedge^{k-1}(E \oplus F)\right)$ generated by all the elementary $k$-vector of the form

$$
e_{i_{1}} \wedge \cdots \wedge e_{i_{h}} \wedge f_{j_{1}} \wedge \cdots \wedge f_{j_{k-h}}
$$

for $1 \leq h \leq k$ and $1 \leq i_{1}<\cdots<i_{h} \leq k, 1 \leq j_{1}<\cdots<j_{k-h} \leq m$ (with no $f$ vectors if $h=k \overline{)}$, is also a subbundle of $\wedge^{k} T_{\Gamma} M$ which is $\wedge^{k} D X_{t}$-invariant.

Moreover, $\widetilde{E} \oplus \widetilde{F}=\wedge^{k} T_{\Gamma} M$ gives a splitting of the $k$ th exterior power of the subbundle $T_{\Gamma} M$. We stress that each subbundle $\widetilde{E}_{x}, \widetilde{F}_{x}$ at any given $x \in \Gamma$ is generated by basis of both $E_{x}$ and $F_{x}$ in a pointwise construction.

Let us assume that $\operatorname{dim} F_{x} \geq 2$ is constant for $x \in \Gamma$ and there are positive constants $C, \lambda$ such that for every $x \in \Gamma$ and every $p$-dimensional linear subspace $L_{x} \subset F_{x}$ one has $\left|\operatorname{det}\left(\left.D X_{-t}\right|_{L_{x}}\right)\right|<C e^{-\lambda t}$, for all $t>0$. That is, $F$ is $p$-sectionally expanding.

By the above discussion, this means that $\left\|\wedge^{k} D X_{-t}\left(u_{1} \wedge \cdots \wedge u_{p}\right)\right\| \leq C e^{-\lambda t}\left\|u_{1} \wedge \cdots \wedge u_{p}\right\|$ for any orthonormal basis $u_{1}, \cdots, u_{p}$ of $F_{x}$.

We are going to show that this inequality for a certain basis implies that $\left\|\wedge^{k} D X_{-t}(w)\right\| \leq$ $C e^{-\lambda t}\|w\|$ for all $w \in \wedge^{k} F_{x}$. Thus, it is enough to obtain the relevant bounds on certain elementary $k$-vectors of the $k$-exterior power extension.

We recall that given a linear isomorphism $A:(E,\langle\cdot, \cdot\rangle) \rightarrow(F,[\cdot, \cdot])$ between finite dimensional Euclidean vector spaces, the Singular Value Decomposition provides:

- $d=\operatorname{dim} E=\operatorname{dim} F$ non-negative reals $0 \leq \lambda_{1} \leq \ldots \leq \lambda_{d}$;

- a pair of orthonormal basis $\mathcal{U}=\left\{u_{1}, \ldots, u_{d}\right\}$ of $E$ and $\mathcal{V}=\left\{v_{1}, \ldots, v_{d}\right\}$ of $F$ such that $A u_{i}=\lambda_{i} v_{i}, i=1, \ldots, d$.

We say that $\mathcal{U}$ is a singular basis and $\lambda_{1}, \ldots, \lambda_{d}$ are the corresponding singular values for the given linear transformation. Let us define $\mathcal{U}^{p}=\left\{u_{i_{1}} \wedge \cdots \wedge u_{i_{p}}: 1 \leq i_{1}<\cdots<i_{p} \leq d\right\}$ for $1<p \leq d$ and observe that $\mathcal{U}^{p}$ is an orthonormal basis for $\wedge^{p} E$ and, likewise, $\mathcal{V}^{p}$ is an orthonormal basis for $\wedge^{p} F$, with respect to the induced exterior inner products. Moreover

$$
\wedge^{p} A \cdot u_{i_{1}} \wedge \cdots \wedge u_{i_{p}}=\left(\lambda_{i_{1}} \cdots \lambda_{i_{p}}\right) \cdot v_{i_{1}} \wedge \cdots \wedge v_{i_{p}}
$$

for all relevant indices, and the number of elements in $\mathcal{U}^{p}$ is $\left(\begin{array}{l}d \\ p\end{array}\right)=\operatorname{dim} \wedge^{p} E$. Hence, $\mathcal{U}^{p}$ is a singular basis for the operator $\wedge^{p} A$ and for each $u \in \mathcal{U}^{p}$ the corresponding singular values are $\lambda(u)=\lambda\left(u_{i_{1}} \wedge \cdots \wedge u_{i_{p}}\right)=\lambda_{i_{1}} \cdots \lambda_{i_{p}}$. 
Remark 3.3. If $E=E_{1} \oplus E_{2}$ and $F=F_{1} \oplus F_{2}$ are orthogonal splittings of $(E,\langle\cdot, \cdot\rangle)$ and $(F,[\cdot, \cdot])$ preserved by $A$, that is, $A\left(E_{i}\right)=F_{i}, i=1,2$, then given singular basis $\mathcal{U}_{i}$ for $\left.A\right|_{E_{i}}, i=1,2$ we have that $\mathcal{U}_{1} \cup \mathcal{U}_{2}$ is a singular basis for $A$. Likewise, $\left(\mathcal{U}_{1} \cup \mathcal{U}_{2}\right)^{p}$ is a singular basis for $\wedge^{p} A$ for any $1<p \leq d$.

Lemma 3.4. Let $A:(E,\langle\cdot, \cdot\rangle) \rightarrow(F,[\cdot, \cdot])$ be a linear isomorphism between d-dimensional Euclidean vector spaces, let $\mathcal{U}$ be a singular basis for $A$ and fix $1<p \leq d$. If there exists $\xi>0$ satisfying $\left\|\wedge^{p} A \cdot u\right\| \leq \xi$ for $u \in \mathcal{U}^{p}$, then $\left\|\wedge^{p} A\right\|=\sup _{w \in \wedge^{p} E} \frac{\left\|\wedge^{p} \overline{A \cdot w}\right\|}{\|w\|} \leq \xi$. Moreover, if $p<d$, then $\left\|\wedge^{p+1} A\right\| \leq \xi\|A\|$.

Proof. Fixing $w \in \wedge^{p} E$ we have $w=\sum_{u \in \mathcal{U}^{p}} \beta(w, u) u$ for some scalars $\beta(w, u)$ so that $\sum_{u \in \mathcal{U}^{p}} \beta(w, u)^{2}=\|w\|^{2}$. Thus, by definition of singular basis

$$
\begin{aligned}
\left\|\wedge^{p} A \cdot w\right\|^{2} & =\left\|\sum_{u \in \mathcal{U}^{p}} \beta(w, u) \cdot\left(\wedge^{p} A \cdot u\right)\right\|^{2}=\sum_{u \in \mathcal{U}^{p}} \beta(w, u)^{2}\left\|\wedge^{p} A \cdot u\right\|^{2} \\
& \leq \sum_{u \in \mathcal{U}^{p}} \beta(w, u)^{2} \xi^{2}=\xi^{2}\|w\|^{2} .
\end{aligned}
$$

Since $w \in \wedge^{p} E$ was arbitrarily chosen, the proof of the first statement of the lemma is complete.

Finally, note that the assumption implies that $\lambda(u) \leq \xi$ for all $u \in \mathcal{U}^{p}$. Then the singular values of $\wedge^{p+1} A$ are products of singular values of $A$ by some $\lambda(u)$ for $u \in \mathcal{U}^{p}$. Since $\lambda_{i} \leq\|A\|$ we get $\left\|\wedge^{p+1} A\right\| \leq\|A\| \cdot\left\|\wedge^{p} A\right\| \leq \xi\|A\|$, completing the proof of the lemma.

3.3. Dominated/partial/sectional-hyperbolic splittings and exterior powers. In [4], the first and last authors together proved the following relation between a dominated splitting and its exterior power.

Theorem 3.5. [4, Theorem A] Assume that $T_{\Gamma} M=E \oplus F$ is a $D X_{t}$ invariant splitting with $\operatorname{dim} F=k \geq 2$. The splitting $T_{\Gamma} M=E \oplus F$ is dominated for $D X_{t}$ if, and only if, $\wedge^{k} T_{\Gamma} M=\widetilde{E} \oplus \widetilde{F}$ is a dominated splitting for $\wedge^{k} D X_{t}$.

Hence, the existence of a dominated splitting $T_{\Gamma} M=E_{\Gamma} \oplus F_{\Gamma}$ over the compact $X_{t^{-}}$ invariant subset $\Gamma$, is equivalent to that the bundle $\wedge^{k} T_{\Gamma} M$ admits a dominated splitting with respect to $\wedge^{k} D X_{t}: \wedge^{k} T_{\Gamma} M \rightarrow \wedge^{k} T_{\Gamma} M$.

As a consequence, they obtain the next characterization of three-dimensional singular sets.

Corollary 3.6. [4, Corollary 1.5] Assume that $T_{\Gamma} M=E \oplus F$ is a $D X_{t}$ invariant splitting. Suppose that $M$ has dimension 3, $E$ is uniformly contracted by $D X_{t}$ and $\operatorname{dim} F=2$. Then $E \oplus F$ is a singular hyperbolic splitting for $D X_{t}$ if, and only if, $\widetilde{E} \oplus \widetilde{F}$ is a partially hyperbolic splitting for $\wedge^{2} D X_{t}$ such that $\widetilde{F}$ is uniformly expanded by $\wedge^{2} D X_{t}$.

The result below generalizes Corollary 3.6 to arbitrary $n$ and $k$. 
Lemma 3.7. [19, Lemma 3.4] Assume that $T_{\Gamma} M=E \oplus F$ is a $D X_{t}$-invariant splitting with $\operatorname{dim} M=n$ and $\operatorname{dim} F=k \geq 2$. The subbundle $F_{\Gamma}$ is volume expanding by $D X_{t}$ if, and only if, $\widetilde{F}$ is uniformly expanded by $\wedge^{k} D X_{t}$.

In particular, $E \oplus F$ is a singular-hyperbolic splitting, where $F$ is volume expanding for $D X_{t}$ if, and only if, $\widetilde{E} \oplus \widetilde{F}$ is a partially hyperbolic splitting for $\wedge^{k} D X_{t}$ such that $\widetilde{F}$ is uniformly expanded by $\wedge^{k} D X_{t}$.

Corollary 3.8. Assume that $T_{\Gamma} M=E \oplus F$ is a $D X_{t}$ invariant splitting. Suppose that $E$ is uniformly contracted by $D X_{t}$. Then $E \oplus F$ is a singular-hyperbolic splitting for $D X_{t}$ if, and only if, $\widetilde{E} \oplus \widetilde{F}$ is a partially hyperbolic splitting for $\wedge^{k} D X_{t}$ such that $\widetilde{F}$ is uniformly expanded by $\wedge^{k} D X_{t}$.

Let $M$ be an $m$-dimensional Riemannian manifold with $\langle\cdot, \cdot\rangle$ inner product in $T_{\Gamma} M$, and $\langle\cdot, \cdot\rangle_{*}$ the inner product in $\wedge^{k} T_{\Gamma} M$ induced by $\langle\cdot, \cdot\rangle$ where $\wedge^{k} T_{\Gamma} M=\bigcup_{x \in \Gamma} \wedge^{k} T_{x} M$. So for $x \in \Gamma$, we have that $\langle\cdot, \cdot\rangle$ is defined on $T_{x} M$, and $\langle\cdot, \cdot\rangle_{*}$ is defined on $\wedge^{k} T_{x} M$.

Lemma 3.9. Let $N$ be a $n$-dimensional vector bundle. Then, for each inner product $[\cdot, \cdot]_{*}$ in $\wedge^{n-1} N$ there exists an inner product $[\cdot, \cdot]$ on $N$ such that $[\cdot, \cdot \cdot]_{*}$ is induced by $[\cdot, \cdot \cdot]$.

Proof. Let $N$ be a $n$-dimensional vector bundle with an inner product $\langle\cdot, \cdot\rangle$ on $N$, and $\langle\cdot, \cdot\rangle_{*}$ the inner product in $\wedge^{n-1} N$ induced by $\langle\cdot, \cdot\rangle$ (see Remark 3.1).

Take $[\cdot, \cdot]_{*}$ an arbitrary inner product in $\wedge^{n-1} N$. Using that $[\cdot, \cdot]_{*}$ and $\langle\cdot, \cdot\rangle_{*}$ are inner products in $\wedge^{n-1} N$ there exists a vector bundle isomorphism $J: \wedge^{n-1} N \rightarrow \wedge^{n-1} N$ such that $[u, v]_{*}=\langle J(u), J(v)\rangle_{*}$ for $u, v \in \wedge^{n-1} N$.

Define $\varphi: G L(N) \rightarrow G L\left(\wedge^{(n-1)} N\right)$ by $A \mapsto \wedge^{n-1} A$ and note that $\varphi$ is an injective linear homomorphism. Hence, due to the dimensions of the spaces, $\varphi$ is a linear isomorphism. Thus, there exists $A \in G L(N)$ such that $\wedge^{n-1} A=J$. This implies that $[u, v]_{*}=\left\langle\wedge^{n-1} A(u), \wedge^{n-1} A(v)\right\rangle_{*}$ for $u, v \in \wedge^{n-1} N$.

We define $[[x, y]]:=\langle A(x), A(y)\rangle$ for $x, y \in N_{z}$ and $z \in \Gamma$. Denote also by $[[\cdot, \cdot]]$ the inner product over $\wedge^{n-1} N$ induced by the inner product $[[\cdot, \cdot]]$ over $N$ (see Remark 3.1). Then if $u=u_{1} \wedge \cdots \wedge u_{n-1}$ and $v=v_{1} \wedge \cdots \wedge v_{n-1}$ we get $[[u, v]]=\operatorname{det}\left(\left[\left[u_{i}, v_{j}\right]\right]\right)_{n-1 \times n-1}=$ $\operatorname{det}\left(\left\langle A u_{i}, A v_{j}\right\rangle\right)_{n-1 \times n-1}$ and so, on the one hand, we obtain

$$
[[u, v]]=\operatorname{det}\left(\left\langle A\left(u_{i}\right), A\left(v_{j}\right)\right\rangle\right)_{n-1 \times n-1}=\left\langle\wedge^{n-1} A(u), \wedge^{n-1} A(v)\right\rangle_{*} .
$$

On the other hand, we also have $[u, v]_{*}=\langle J(u), J(v)\rangle_{*}=\left\langle\wedge^{n-1} A(u), \wedge^{n-1} A(v)\right\rangle_{*}$. Therefore, $[\cdot, \cdot]_{*}=[[\cdot, \cdot]]$ on all $(n-1)$-vectors and so by bilinearity equality holds for all elements of $\wedge^{n-1} N$ and we are done.

\section{Proofs of MAIN RESUlts}

We are now able to prove our main theorems.

4.1. Existence of singular adapted metric. Here we prove Theorem A.

Let $\Gamma$ be a compact invariant subset of $X$ admitting a singular-hyperbolic splitting $T_{\Gamma} M=E^{s} \oplus E^{c}$ with rate given by the constant $\lambda>0$; see Definitions 1,2 and 3 . 
Let $Y$ be the north-pole-south-pole vector field on the one-sphere $\mathbb{S}^{1}$, which we view as $\mathbb{S}^{1}=\mathbb{R} / \mathbb{Z}$, with one sink (the south pole, the point $S=0 \in \mathbb{S}^{1}$ ) and one source (the north pole) and no other recurrent points. Thus $Y$ can be seen as a periodic smooth function $f_{0}: \mathbb{S}^{1} \rightarrow \mathbb{R}$

We take the skew-product vector field $Z$ on $M \times \mathbb{S}^{1}$ given by $(x, \theta) \mapsto(X(x), \mu(x) Y(\theta)) \in$ $T_{x} M \times T_{t} \mathbb{S}^{1}$, where $\mu: M \rightarrow \mathbb{R}$ is a smooth function to be specified. Let $Z_{t}: M \times \mathbb{S}^{1} \circlearrowleft$ be the flow generated by $Z$ and consider the compact $Z$-invariant subset $\widetilde{\Gamma}=\Gamma \times\{S\}$.

Note that $T_{\widetilde{\Gamma}}\left(M \times \mathbb{S}^{1}\right)=E^{s} \oplus F \oplus E^{c}$ where $F=T_{S} \mathbb{S}^{1}$ is a one-dimensional subspace, $\operatorname{dim} E^{s}=d_{s}$ and $\operatorname{dim} E^{c}=d_{c}, d_{s}+d_{c}=m$. Indeed, the flow $(x, \theta, t) \mapsto Z_{t}(X, \theta)=$ $\left(X_{t}(x), Y_{t, x}(\theta)\right)$, where

$$
Y_{t, x}(\theta)=\theta+\int_{0}^{t} \mu\left(X_{s}(x)\right) f_{0}\left(Y_{s, x}(\theta)\right) d s, \quad(x, \theta) \in M \times \mathbb{S}^{1}, t \in \mathbb{R},
$$

is uniquely defined and preserves $\{0\} \times T \mathbb{S}^{1}$. In fact, any curve $v(s)=\{x\} \times \theta(t)$ for a given fixed $x \in M$ and smooth $\theta: I \rightarrow \mathbb{S}^{1}$ is sent to $Z_{t}(v(s))=\left(X_{t}(x), Y_{t, x}(\theta(s))\right)$, which is again a curve in $\left\{X_{t}(x)\right\} \times \mathbb{S}^{1}$; where $I$ denotes the unit interval $[0,1]$.

Denoting by $[\cdot, \cdot]$ an inner product over $T_{\Gamma} M$ and by $\cdot$ some inner product on $\mathbb{S}^{1}$, we define for $v=v_{1}+f_{1}, u=u_{1}+f_{2} \in E^{s} \oplus F \oplus E^{c}$, where $v_{1}, u_{1} \in E^{s} \oplus E^{c}$ and $f_{1}, f_{2} \in F$, the inner product on $T_{\Gamma \times\{S\}}\left(M \times \mathbb{S}^{1}\right)$ by $\langle v, u\rangle=\left[v_{1}, u_{1}\right]+f_{1} \cdot f_{2}$.

Remark 4.1. (1) The choice of the inner product ensures that the subbundle $F$ becomes orthogonal to $E^{s} \oplus E^{c}$.

(2) We also have that $\left\|\left.D Z_{t}(x, S)\right|_{F}\right\|=\exp \left[-\alpha_{0} \int_{0}^{t} \mu\left(X_{s}(x)\right) d s\right]=\mathrm{m}\left(\left.D Z_{t}(x, S)\right|_{F}\right)^{2}$, $x \in M, t \in \mathbb{R}$, where $-\alpha_{0}=f_{0}^{\prime}(0)<0$, for any Finsler on $M \times\{S\}$, by construction of $Z_{t}$.

We are going to show that there exists the dominated splitting $T_{\widetilde{\Gamma}}\left(M \times \mathbb{S}^{1}\right)=E^{s} \oplus\left(F \oplus E^{c}\right)$ over the compact invariant set $\widetilde{\Gamma}$ of $Z$. To prove this result we use the following auxiliary results.

4.1.1. Subadditive functions of the orbits of a flow and exponential growth. We say that a family of functions $\phi: \mathbb{R} \times \Gamma \rightarrow \mathbb{R}$ is subadditive if

$$
\phi(t+s, x) \leq \phi\left(s, X_{t}(x)\right)+\phi(t, x), \quad \text { for all } t, s \in \mathbb{R}, x \in \Gamma .
$$

Lemma 4.2. [2, Lemma 4.12] Let $\phi: \mathbb{R} \times \Gamma \rightarrow \mathbb{R}$ be a subadditive function for the flow of $X$ satisfying $\phi(0, x)=0$ and $D(x):=\lim _{\sup _{h \rightarrow 0}}(\phi(h, x) / h)<\infty$. Then $\left.\partial_{h} \phi(h, x)\right|_{h=0}=$ $D(x)=\lim _{h \rightarrow 0} \phi(h, x) / h$.

We say that $D(x)$ is the infinitesimal generator of $\phi$ and we can deduce the following useful relation; see e.g. [2, Section 4.3.1.1]:

$$
\phi(t, x)=\int_{0}^{t} D\left(X_{s}(x)\right) d s, \quad x \in \Gamma, t \in \mathbb{R} .
$$

\footnotetext{
${ }^{2}$ Here $\mathrm{m}(A)=\inf _{\|u\|=1}\|A(u)\|$ for a morphism $A$ of normed vector spaces.
} 
Hence, for each fixed $x \in \Gamma$, the function of $t$ above is in fact additive. The following will be very useful.

Lemma 4.3. If $\phi, \psi:[0,+\infty) \times \Gamma \rightarrow \mathbb{R}$ be subadditive functions on an $X$-invariant compact subset $\Gamma$ such that there exists $a>0$ satisfying $\phi(t, x) \leq \psi(t, x)$ for all $0 \leq t \leq a$ and $x \in \Gamma$. Then $\phi \leq \psi$.

Proof. For any given $s \geq 0$ we can write $s=[s]+t$ with $[s]=\sup \left\{n \in \mathbb{Z}^{+}: n a \leq s\right\}$ and $0 \leq t \leq a$. Then let $m=[s] \in \mathbb{Z}$ and by assumption

$$
\phi(t, x)=\int_{0}^{t} D\left(X_{u} x\right) d u \leq \int_{0}^{t} E\left(X_{u} x\right) d u=\psi(t, x), \quad x \in \Gamma
$$

where $D, E$ are the infinitesimal generators of $\phi, \psi$. Hence for any fixed $x \in \Gamma$

$$
\begin{aligned}
\phi(s, x) & =\int_{0}^{s} D\left(X_{u} x\right) d u=\sum_{i=0}^{(n-1) a} \int_{0}^{a} D\left(X_{i a+u} x\right) d u+\int_{n}^{t} D\left(X_{n a+u} x\right) d u \\
& \leq \sum_{i=0}^{(n-1) a} \int_{0}^{a} E\left(X_{i a+u} x\right) d u+\int_{n a}^{t} E\left(X_{n a+u} x\right) d u=\int_{0}^{s} E\left(X_{u} x\right) d u=\psi(s, x)
\end{aligned}
$$

as stated.

We apply this to $\phi(t, v)=\log \left\|D Z_{t} \cdot v\right\|$ for $v \in T^{1}\left(M \times \mathbb{S}^{1}\right)$ obtaining a continuous function $D^{s}: T_{\widetilde{\Gamma}}^{1}\left(M \times \mathbb{S}^{1}\right) \cap E^{s} \rightarrow \mathbb{R}$, where $T^{1}$ denotes the unit tangent bundle, such that

$$
\log \left\|D Z_{t} v_{s}\right\|=\int_{0}^{t} D^{s}\left(\phi_{u} v_{s}\right) d u, \quad \text { with } \quad \phi_{u} v_{s}=\frac{D Z_{u} v_{s}}{\left\|D Z_{u} v_{s}\right\|} \quad \text { and } \quad v_{s} \in E_{x}^{s},\left\|v_{s}\right\|=1
$$

see [1, Theorem 1.11]. Analogously we obtain continous functions $D^{c}, D_{F}^{s}$ on $T_{\widetilde{\Gamma}}^{1}\left(M \times \mathbb{S}^{1}\right)$ satisfying

$$
\begin{aligned}
\log \left\|D Z_{-t} \phi_{t} v_{c}\right\| & =\int_{0}^{t} D^{c}\left(\phi_{u} v_{c}\right) d u \quad \text { with } \quad v_{c} \in E_{x}^{c},\left\|v_{c}\right\|=1 ; \quad \text { and } \\
\log \left\|D Z_{t} v\right\| & =\int_{0}^{t} D_{F}^{s}\left(\phi_{u} v\right) d s \quad \text { with } \quad v \in\left(E^{s} \oplus F\right)_{x},\|v\|=1 .
\end{aligned}
$$

Note that in Remark 4.1(2) we already obtained an expression for the infinitesimal generator along the one-dimensional bundle $F$.

4.1.2. Dominated splitting for the skew-product flow. We are now ready to prove the following result.

Lemma 4.4. There exist $C, \xi>0$ and a smooth function $\mu: M \rightarrow \mathbb{R}$ such that for the skew-product vector field $Z$ on $M \times \mathbb{S}^{1}$, for all $x \in \widetilde{\Gamma}$ and $t>0$

(1) $\left\|\left.D Z_{t}\right|_{E_{x}^{s}}\right\| \cdot\left\|\left.D Z_{-t}\right|_{F \oplus E_{Z_{t} x}^{c}}\right\| \leq C e^{-\xi t}$; and

(2) $\left\|\left.D Z_{t}\right|_{E_{x}^{s} \oplus F}\right\| \cdot\left\|\left.D Z_{-t}\right|_{E_{Z_{t}}^{c}}\right\| \leq C e^{-\xi t}$;

where $\|w\|=\langle w, w\rangle^{1 / 2}, w \in T_{\widetilde{\Gamma}}\left(M \times \mathbb{S}^{1}\right)$. 
Proof of Lemma 4.4. For the first item, take $v_{s} \in E_{x}^{s}$ and $w=f+v_{c} \in\left(F \oplus E^{c}\right)_{Z_{t} x}$, where $f \in F_{Z_{t} x}$ and $v_{c} \in E_{Z_{t} x}^{c}$ and all these vectors are unitary. Then

$$
\begin{aligned}
& \left\|D Z_{t}\left(v_{s}\right)\right\|^{2} \cdot\left\|D Z_{-t}\left(f+v_{c}\right)\right\|^{2}=\left\|D Z_{t}\left(v_{s}\right)\right\|^{2} \cdot\left(\left\|D Z_{-t}(f)\right\|^{2}+\left\|D Z_{-t}\left(v_{c}\right)\right\|^{2}\right) \\
& \quad=\left\|D Z_{t}\left(v_{s}\right)\right\|^{2} \cdot\left\|D Z_{-t}(f)\right\|^{2}+\left\|D Z_{t}\left(v_{s}\right)\right\|^{2} \cdot\left\|D Z_{-t}\left(v_{c}\right)\right\|^{2} \\
& \quad=\left\|D Z_{-t}(f)\right\|^{2} \exp \left[2 \int_{0}^{t} D^{s} \phi_{u} v_{s} d u\right]+\exp \left[2 \int_{0}^{t}\left(D^{s} \phi_{u} v_{s}+D^{c} \phi_{u} v_{c}\right) d u\right] \\
& \quad=e^{-2 \int_{0}^{-t} \alpha_{0} \mu \circ Z_{u}\left(Z_{t} x\right) d s+2 \int_{0}^{t} D^{s} \cdot \phi_{u} v_{s} d u}+e^{2 \int_{0}^{t}\left(D^{s} \cdot \phi_{u} v_{s}+D^{c} \cdot \phi_{u} v_{c}\right) d u} \\
& \quad \leq e^{2 \int_{0}^{t} D^{s} \cdot \phi_{u} v_{s} d u}\left(e^{2 \int_{0}^{t} \alpha_{0} \mu \circ Z_{u} x d u}+e^{\left.2 \int_{0}^{t} D^{c} \cdot \phi_{u} v_{c}\right) d u}\right) \\
& \quad=\exp \left[2 \int_{0}^{t}\left(D^{s} \cdot \phi_{u} v_{s}+D^{c} \cdot \phi_{u} v_{c}\right) d u\right]+\exp \left[2 \int_{0}^{t}\left(D^{s} \cdot \phi_{u} v_{s}+\alpha_{0} \mu \circ Z_{u} x\right) d u\right] .
\end{aligned}
$$

Now note that from domination we get

$$
\begin{aligned}
\exp \left[2 \int_{0}^{t}\left(D^{s} \cdot \phi_{u} v_{s}+D^{c} \cdot \phi_{u} v_{c}\right) d u\right] & =\left\|D Z_{t} \cdot v_{s}\right\|^{2} \cdot\left\|D Z_{-t} \phi_{t} v_{c}\right\|^{2} \\
& \leq\left\|D Z_{t}\left|E_{x}^{s}\left\|^{2} \cdot\right\| D Z_{-t}\right| E_{X_{t} x}^{c}\right\|^{2} \leq K e^{-2 \lambda t} .
\end{aligned}
$$

So, it is enough to choose $\mu$ so that $\int_{0}^{t}\left(D^{s} \phi_{u} v_{s}+\alpha_{0} \mu \circ Z_{s} x\right) d s \leq-\xi t$ for some $\xi>0$ and all choices of unitary vectors $v_{s} \in E_{x}^{s}$. If $\mu: \widetilde{\Gamma} \rightarrow \mathbb{R}$ is a smooth function such that

$$
\sup _{\left\|v_{s}\right\|=1} D^{s}\left(v_{s}\right)+\zeta<-\alpha_{0} \mu(x)<\sup _{\left\|v_{s}\right\|=1} D^{s}\left(v_{s}\right)+2 \zeta, \quad x \in \widetilde{\Gamma}
$$

and $\zeta>0$ is sufficiently small, then $\int_{0}^{t}\left(D^{s} \phi_{u} v_{s}+\alpha_{0} \mu\left(Z_{u} x\right)\right) d u \leq-\zeta t$. This proves item (1) with $C=K, \xi=\zeta$ and $\mu$ chosen as above.

For the second item, note that for all $t>0, v_{s} \in E_{x}^{s}$ with $\left\|v_{s}\right\|=1$ and $x \in \widetilde{\Gamma}$

$$
\log \left\|\left.D Z_{t}\right|_{F}\right\|=\int_{0}^{t}-\alpha_{0} \mu\left(Z_{u} x\right) d u>\int_{0}^{t}\left(D^{s} \phi_{u} v_{s}+\zeta\right) d u=\zeta t+\log \left\|D Z_{t} v_{s}\right\|
$$

which implies

$$
\log \left\|\left.D Z_{t}\right|_{F}\right\| \geq \zeta t+\log \left\|D Z_{t} v_{s}\right\| \quad \text { and } \quad\left\|\left.D Z_{t}\right|_{\left(E^{s} \oplus F\right)_{x}}\right\|=\left\|\left.D Z_{t}\right|_{F}\right\| .
$$

Hence we obtain $D_{F}^{s}=-\alpha_{0} \mu$. Thus

$$
\begin{aligned}
\left\|\left.D Z_{t}\right|_{\left(E^{s} \oplus F\right)_{x}}\right\| \cdot\left\|\left(\left.D Z_{t}\right|_{E_{x}^{c}}\right)^{-1}\right\| & =\left\|\left.D Z_{t}\right|_{F}\right\| \cdot\left\|\left.D Z_{-t}\right|_{E_{Z_{t} x}^{c}}\right\| \\
& \leq \exp \left[\int_{0}^{t}\left(-\alpha_{0} \mu\left(Z_{u} x\right)+\sup _{\left\|v_{c}\right\|=1} D^{c} \phi_{u} v_{c}\right) d u\right] \\
& \leq \exp \left[2 \zeta t+\int_{0}^{t}\left(\sup _{\left\|v_{s}\right\|=1} D^{s} \phi_{u} v_{s}+\sup _{\left\|v_{c}\right\|=1} D^{c} \phi_{u} v_{c}\right) d u\right] \\
& \leq e^{2 \zeta t}\left\|\left.D Z_{t}\right|_{E_{x}^{s}}\right\| \cdot\left\|\left.D Z_{-t}\right|_{E_{Z_{t^{x}}}^{c}}\right\| \leq e^{2 \zeta t} K e^{-\lambda t} .
\end{aligned}
$$


We just have to choose $0<2 \zeta<\lambda$ to conclude the statement of item (2) with $\xi=\lambda-2 \zeta$ and $C=K$.

This concludes the the proof of the Lemma 4.4 with $C=K$ and $\xi=\min \{\zeta, \lambda-2 \zeta\}$.

This shows that there exists the dominated splitting $T_{\widetilde{\Gamma}}\left(M \times \mathbb{S}^{1}\right)=E^{s} \oplus\left(F \oplus E^{c}\right)$ over the compact invariant set $\widetilde{\Gamma}$ of $Z$. In particular, this also shows that $F \oplus E^{c}$ is a partially hyperbolic splitting with respect to $D Z_{t} \mid\left(F \oplus E^{c}\right)$, such that the volume along $E^{c}$ is uniformly expanded. Thus, $F \oplus E^{c}$ is a codimension-one singular-hyperbolic splitting of the subbundle $F \oplus E^{c}$ of $T_{\widetilde{\Gamma}}\left(M \times \mathbb{S}^{1}\right)$.

Remark 4.5. We can write $\wedge^{d_{c}}\left(E^{s} \oplus F \oplus E^{c}\right)=\widetilde{E} \oplus\left[F \wedge\left(\wedge^{d_{c}-1} E^{c}\right)\right] \oplus \wedge^{d_{c}} E^{c}$, where $\widetilde{E}$ is formed by non-null exterior vectors involving always some vector from $E^{s}$.

We denote $\widetilde{F}=F \wedge\left(\wedge^{d_{c}-1} E^{c}\right)$ and $\widetilde{E^{c}}=\wedge^{d_{c}} E^{c}$ in what follows. Then from Theorem 3.5 the splitting $T_{\widetilde{\Gamma}}\left(M \times \mathbb{S}^{1}\right)=E^{s} \oplus\left(F \oplus E^{c}\right)$ is dominated for $D Z_{t}$ if, and only if, $\widetilde{E} \oplus \widetilde{F} \oplus \widetilde{E^{c}}$ is a dominated splitting for $\wedge^{d_{c}} D Z_{t}$. Since we additionally have $\left|\operatorname{det}\left(\left.D Z_{-t}\right|_{E_{x}^{c}}\right)\right| \leq C e^{-\xi t}$ for some $C, \xi>0$ by assumption of singular-hyperbolicity, then $\widetilde{F} \oplus \widetilde{E^{c}}$ is a partially hyperbolic splitting of the invariant subbundle $\wedge^{d_{c}}\left(F \oplus E^{c}\right)$ such that $\widetilde{E^{c}}$ is uniformly expanded by $\left.\wedge^{d_{c}} D Z_{t}\right|_{\widetilde{F} \oplus \widetilde{E^{c}}}$.

Hence, from [10, Theorem 1], there exists an adapted inner product $[\cdot, \cdot]$ for $\left.\wedge^{d_{c}} D Z_{t}\right|_{\widetilde{F} \oplus \widetilde{E^{c}}}$ over $\widetilde{\Gamma}$, that is, there exists $\sigma>0$ satisfying

$$
\left[\left.\wedge^{d_{c}} D Z_{t}\right|_{\widetilde{F}_{x}}\right] \cdot\left[\left.\wedge^{d_{c}} D Z_{-t}\right|_{{\widetilde{E^{c}}}_{Z_{t}(x)}}\right] \leq e^{-\sigma t} \quad \text { and } \quad\left[\left.\wedge^{d_{c}} D Z_{-t}\right|_{{\widetilde{E^{c}}}_{x}}\right] \leq e^{-\sigma t}, \quad \forall t>0, \forall x \in \widetilde{\Gamma} \text {. }
$$

Lemma 3.9 now ensures that there exists an inner product $[[\cdot, \cdot]]$ on the subbundle $F \oplus E^{c}$ such that $[\cdot, \cdot]$ is induced by $[[\cdot, \cdot]]$. This last inner product satisfies the conditions of the following result, whose proof we postpone to the end of this section.

Lemma 4.6. Suppose that there exists an inner product $\langle\langle\cdot, \cdot\rangle\rangle$ on an invariant subbundle $G$ of dimension $m$ of $T_{\Gamma} M$, such that it admits a continuous splitting $G=E \oplus F$ and whose induced inner product $\langle\langle\cdot, \cdot\rangle\rangle_{*}$ on $\wedge^{(m-1)} G$ satisfies for each $x \in \Gamma$ and $t>0$

$$
\left\|\left.\wedge^{m-1} D X_{t}\right|_{\tilde{E}_{x}}\right\|_{*} \cdot\left\|\left.\wedge^{m-1} D X_{-t}\right|_{\tilde{F}_{X_{t}(x)}}\right\|_{*} \leq e^{-\lambda t} \quad \text { and } \quad\left\|\left.\wedge^{m-1} D X_{t}\right|_{\widetilde{F}_{x}}\right\|_{*} \geq e^{\lambda t}
$$

where $\|\cdot\|$ is the norm induced by $\langle\langle\cdot, \cdot\rangle\rangle$. Then there exists an inner product $\langle\cdot, \cdot\rangle$ in $T_{\Gamma} M$ such that for all $t>0$

(1) $\left.\left|D X_{t}\right|_{E_{x}}|\cdot| D X_{-t}\right|_{F_{X_{t}(x)}} \mid \leq e^{-\lambda t}$;

(2) $\left.\left.\left|\wedge^{m-1} D X_{t}\right|_{\tilde{E}_{x}}\right|_{*} \cdot\left|\wedge^{m-1} D X_{-t}\right|_{\tilde{F}_{X_{t}(x)}}\right|_{*} \leq e^{-\lambda t}$; and

(3) $\left.\left|\wedge^{m-1} D X_{t}\right|_{\widetilde{F}_{x}}\right|_{*} \geq e^{\lambda t}$.

where $|\cdot|$ is the norm induced by $\langle\cdot, \cdot\rangle$.

Hence, applying the above lemma to the subbubdle $G=F \oplus E^{c}$ with $F=E^{c}$ and $E=F$, we obtain that there exists a singular adapted inner product $[[\cdot, \cdot]]_{*}$ on $F \oplus E^{c}$, that is, satisfying

$$
\left[\left[\left.D Z_{t}\right|_{F_{x}}\right]\right]_{*} \cdot\left[\left[\left.D Z_{-t}\right|_{E_{Z_{t}(x)}^{c}}\right]\right]_{*} \leq e^{-\sigma t} \quad \text { and } \quad\left[\left[\left.\wedge^{d_{c}} D Z_{-t}\right|_{\widetilde{E}^{c} x}\right]\right]_{*} \leq e^{-\sigma t}
$$


for some $\sigma>0$ and for all $t>0, x \in \widetilde{\Gamma}$.

4.1.3. Adapting the domination on the stable direction. We need the following lemma to find a metric which is adapted to the uniform contraction along the stable direction.

Lemma 4.7. Let $H=E^{s} \oplus F \oplus E^{c}$ be a $D Z_{t}$-invariant and continuous splitting of a vector subbundle such that $E^{s}$ is uniformly $(K, \lambda)$-contracted; the subbundle $F \oplus E^{c}$ admits an inner product $\langle\cdot, \cdot\rangle$ inducing a metric $|\cdot|$ satisfying items (1), (2) and (3) of the conclusion of Lemma 4.6; and F satisfies Remark 4.1 and Lemma 4.4. Then there exists a singular adapted metric for the subbundle $E^{s} \oplus E^{c}$.

Proof. We follow [10] and define a norm on $E^{s}$ by $\left|u_{s}\right|=\int_{0}^{+\infty} e^{\beta \xi r}\left\|D X_{\beta r} u_{s}\right\| d r$ for any $u_{s} \in E^{s}$, where $\xi$ is given by Lemma 4.4 and $\beta=K /(\lambda-2 \xi)$. It is easy to check that $\left|D X_{t} u_{s}\right| \leq e^{-\xi t}\left|u_{s}\right|$ for all $t>0$ and also that this norm is Riemannian, i.e., induced by an inner product $(\cdot, \cdot)$.

From the construction of $F$ and (4.2) we obtain for $v \in E_{x}^{s}$

$$
\begin{aligned}
\left|D X_{t} v\right| & =\int_{0}^{\infty} e^{\beta \xi u}\left\|D X_{\beta u+t} v\right\| d u \leq \int_{0}^{\infty} e^{\beta \xi u} e^{-\xi(\beta u+t)}\left\|\left.D Z_{\beta u+t}\right|_{F_{x}}\right\| d u \\
& =e^{-\xi t} \int_{t / \beta}^{\infty}\left\|\left.D Z_{\beta r}\right|_{F_{x}}\right\| d r .
\end{aligned}
$$

We need an auxiliary result which we state here but whose proof we postpone.

Lemma 4.8. There exists $a>0$ so that $e^{-\xi t} \int_{t / \beta}^{\infty}\left\|\left.D Z_{\beta u}\right|_{F_{x}}\right\| d u \leq\left\|D Z_{t} \mid F_{x}\right\|$ for all $0 \leq t \leq a$.

Hence we deduce that $\left|D X_{t} v\right| \leq\left\|D Z_{t} \mid F_{x}\right\|$ for each $0 \leq t \leq a, x \in \widetilde{\Gamma}$ and some $a>0$. By Lemma 4.3 we conclude that the inequality can be extended to all $t \geq 0$. Since the norm of $\left.D Z_{t}\right|_{F_{x}}$ does not depend on the chosen Finsler, we can write

$$
\begin{aligned}
\left|D X_{t}\right|_{E_{x}^{s}} \mid \cdot\left[\left[\left.D Z_{-t}\right|_{E_{Z_{t}(x)}^{c}}\right]\right]_{*} & \leq\left\|\left.D Z_{t}\right|_{F_{x}}\right\| \cdot\left[\left[\left.D Z_{-t}\right|_{E_{Z_{t}(x)}^{c}}\right]\right]_{*} \\
& =\left[\left[\left.D Z_{t}\right|_{F_{x}}\right]\right]_{*} \cdot\left[\left[\left.D Z_{-t}\right|_{E_{Z_{t}(x)}^{c}}\right]\right]_{*} \leq e^{-\sigma t} .
\end{aligned}
$$

We can now define an inner product $\langle\cdot, \cdot\rangle$ on $T_{\Gamma} M=E^{s} \oplus E^{c}$ by $\langle u, v\rangle=\left(u_{s}, v_{s}\right)+\left[\left[u_{c}, v_{c}\right]\right]_{*}$ for all $u=u_{s}+u_{c}, v=v_{s}+v_{c} \in T_{\Gamma} M$, and let $\langle\cdot, \cdot\rangle_{*}$ be the induced inner product by $\langle\cdot, \cdot\rangle$ on $\wedge^{d_{c}} T_{\Gamma} M$.

Then $\langle\cdot, \cdot\rangle$ is an inner product in $T_{\Gamma} M$ adapted to the partially hyperbolic splitting $E^{s} \oplus E^{c}$ for $D X_{t}$. Moreover, from the definition of the inner product and exterior power, it follows that $\left|\operatorname{det}\left(D X_{-t} \mid E_{x}^{c}\right)\right| \leq e^{-\sigma t}$ for all $t>0$.

We have obtained a singular adapted metric for $E^{s} \oplus E^{c}$ finishing the proof of Lemma 4.7.

This completes the proof of Theorem A except for the proof of Lemma 4.6 and Lemma 4.8, which we present next. 
Proof of Lemma 4.6. Let $u \in E_{x}$ and $v \in F_{X_{t}(x)}$ be such that $\|u\|=1=\|v\|$. We observe that for a given fixed $t \in \mathbb{R}$

$$
\left\|D X_{t} u\right\| \cdot\left\|D X_{-t} v\right\|=\left\|\wedge^{k} D X_{t}\left(u \wedge u_{2} \wedge \cdots \wedge u_{k}\right)\right\| \cdot\left\|\wedge^{k} D X_{-t}\left(v \wedge v_{2} \wedge \cdots \wedge v_{k}\right)\right\|
$$

if we choose $u_{2}, \cdots, u_{k} \in E_{x}$ and $v_{2}, \cdots, v_{k} \in F_{X_{t}(x)}$ such that:

- $\left\langle D X_{t} u, D X_{t} u_{j}\right\rangle=0$ for $2 \leq j \leq k$ and $\left\langle D X_{t} u_{j}, D X_{t} u_{l}\right\rangle=\delta_{j l}$ for $2 \leq j, l \leq k$;

- $\left\langle D X_{-t} v, D X_{-t} v_{j}\right\rangle=0$ for $2 \leq j \leq k$ and $\left\langle D X_{-t} v_{j}, D X_{-t} v_{l}\right\rangle=\delta_{j l}$ for $2 \leq j, l \leq k$.

Consequently we obtain

$$
\begin{aligned}
\left\|D X_{t} u\right\| \cdot\left\|D X_{-t} v\right\| & \leq\left\|\wedge^{k} D X_{t}\right\|\left\|\wedge^{k} D X_{-t}\right\|\left\|u \wedge u_{2} \wedge \cdots \wedge u_{k}\right\| \cdot\left\|v \wedge v_{2} \wedge \cdots \wedge v_{k}\right\| \\
& \leq e^{-\lambda t}\left\|u \wedge u_{2} \wedge \cdots \wedge u_{k}\right\| \cdot\left\|v \wedge v_{2} \wedge \cdots \wedge v_{k}\right\| .
\end{aligned}
$$

We note that $\left\|u_{j}\right\| \leq\left\|D X_{-t}(x)\right\|$ since $\left\|D X_{t} u_{j}\right\|=1$ and analogously $\left\|v_{j}\right\| \leq\left\|D X_{t}\left(X_{t} x\right)\right\|$ since $\left\|D X_{-t} v_{j}\right\|=1$ for $2 \leq j \leq k$ with $\|u\|=\|v\|=1$.

We now set $R=\max \left\{1, \kappa_{1}\right\}$, where

$$
\kappa_{1}=\sup _{t \in[-1,1]} \sup _{x \in \Gamma}\left\|D X_{t}(x)\right\|
$$

and define $B[0, R]=\{\eta \in T \Gamma:|\eta| \leq R\}$ a compact subset of $T \Gamma$.

Note that if we set $t \in[-1,1]$, then we get $u, u_{2}, \cdots, u_{k}, v, v_{2}, \cdots, v_{k} \in B[0, R]$ in the argument above.

Moreover $\prod_{i=1}^{k} B[0, R]$ is a compact subset of $\prod_{i=1}^{k} T \Gamma=\sum_{p \in \Gamma} T_{p} \Gamma \times \stackrel{k}{k} \times T_{p} \Gamma$ and let $\mathcal{I}: \prod_{i=1}^{k} T \Gamma \rightarrow \wedge^{k} T \Gamma$ be the natural injection given by

$$
\left(w_{1}, \cdots, w_{k}\right) \mapsto w_{1} \wedge \cdots \wedge w_{k}
$$

We define $|\cdot|=\gamma\|\cdot\|\left(\right.$ or $\left.\langle\cdot, \cdot\rangle=\gamma^{2}[[\cdot, \cdot]]\right)$ where $\gamma$ is a positive number such that

$$
\sup _{w \in \prod_{i=1}^{k} B[0, R]}\|\mathcal{I}(w)\| \leq \gamma^{-1} .
$$

It follows that

$$
\begin{aligned}
\left|D X_{t} u\right| \cdot\left|D X_{-t} v\right| & =\gamma\left\|\wedge^{k} D X_{t}\left(u \wedge u_{2} \wedge \cdots \wedge u_{k}\right)\right\| \cdot \gamma\left\|\wedge^{k} D X_{-t}\left(v \wedge v_{2} \wedge \cdots \wedge v_{k}\right)\right\| \\
& \leq e^{-\lambda t} \gamma\left\|u \wedge u_{2} \wedge \cdots \wedge u_{k}\right\| \cdot \gamma\left\|v \wedge v_{2} \wedge \cdots \wedge v_{k}\right\| \leq e^{-\lambda t}
\end{aligned}
$$

and note that the choice of $\gamma$ does not change any of the previous relations involving $\|\cdot\|$. Then for any given fixed $t \in[-1,1]$ we have obtained an adapted metric $|\cdot|$ that satisfies the statement of Lemma 4.6. For general $t>0$ the inequality follows by Lemma 4.3 (with $a=1$ ), since the previous inequality holds between subadditive functions.

We have obtained a metric $|\cdot|$ satisfying item (1) in the statement of Lemma 4.6. Analogously, it satisfies items (2) and (3) of the statement of the Lemma, and we are done. 
Proof of Lemma 4.8. Let us set $f(t)=e^{-\xi t} \int_{t / \beta}^{\infty}\left\|\left.D X_{\beta u}\right|_{F_{x}}\right\| d u$ and use (4.1) to estimate

$$
\begin{aligned}
0 \leq f(t) & \leq e^{-\xi t} \int_{t / \beta}^{\infty} \exp \left(\int_{0}^{\beta u}\left[\sup _{\left\|v_{s}\right\|=1} D^{s}\left(\phi_{w} v_{s}\right)+2 \xi\right] d w\right) d u \\
& \leq e^{-\xi t} \int_{t / \beta}^{\infty} e^{2 \xi \beta u}\left\|D X_{\beta u} \mid E_{x}^{s}\right\| d u \leq e^{-\xi t} \int_{t / \beta}^{\infty} K e^{\beta(2 \xi-\lambda) u} d u \\
& \leq e^{-\xi t} \frac{K e^{(2 \xi-\lambda) t}}{\beta(\lambda-2 \xi)}=\frac{K}{\beta(\lambda-2 \xi)} \leq 1,
\end{aligned}
$$

as long as $\beta \geq K /(\lambda-2 \xi)>0$. Then it is easy to see that

$$
\begin{aligned}
f^{\prime}(t) & =-\xi f(t)-e^{-\xi t}\left\|\left.D X_{t}\right|_{F_{x}}\right\|<0, \forall t>0, \quad \text { and } \\
\left(\frac{f(t)}{\left\|\left.D X_{t}\right|_{F_{x}}\right\|}\right)^{\prime} & =\frac{\left\|\left.D X_{t}\right|_{F_{x}}\right\| \cdot f^{\prime}(t)+f(t) \cdot \alpha_{0} \mu\left(X_{t} x\right)}{\left\|\left.D X_{t}\right|_{F_{x}}\right\|^{2}}
\end{aligned}
$$

so for $t=0$ the last expression is bounded from above by $-1+f(0)\left(\alpha_{0} \mu(x)-\xi\right)$. Since $\mu$ is bounded on $\widetilde{\Gamma}$ (because $D^{s}$ is continuous on the compact set $E^{s} \cap T_{\Gamma}^{1} M$ ), we can choose $\alpha_{0}>0$ small enough so that the last expression is strictly negative for every $x \in \widetilde{\Gamma}$.

Finally, because $f(0) \leq 1$, by smoothness and compactness we conclude that there exists $a>0$ such that $f(t) \leq\left\|D X_{t} \mid F_{x}\right\|$ for all $0 \leq t \leq a, x \in \widetilde{\Gamma}$, completing the proof.

\subsection{The uniformly hyperbolic setting. Here we prove Theorem B.}

Let $\Gamma$ be a uniformly hyperbolic compact invariant subset for the $C^{1}$ vector field $X$ and let $\|\cdot\|$ be an adapted metric, that is, we have (1.2) with $K=1$ and we can assume without loss of generality that $E^{s}, E^{X}$ and $E^{u}$ are orthogonal; see e.g. [10].

We define $|s \cdot X(x)|=|s|$ for all $s \in \mathbb{R}$ and $x \in \Gamma$, which gives a new norm on the one-dimensional line bundle $E^{X}$ such that (recall that $\Gamma$ does not contain singularities: $X(x) \neq \overrightarrow{0}, x \in \Gamma)$

$$
\left|D X_{t}\right| E_{x}^{X} \mid=\frac{\left|D X_{t} X(x)\right|}{|X(x)|}=\frac{\left|X\left(X_{t}(x)\right)\right|}{|X(x)|}=1, \quad x \in \Gamma, t \in \mathbb{R} .
$$

Now we redefine $|u+s \cdot X(x)+v|_{*}^{2}=\|u\|^{2}+|s \cdot X(x)|^{2}+\|v\|^{2}, u \in E_{x}^{s}, v \in E_{x}^{u}, s \in \mathbb{R}$ and note that (1.2) still holds with the same $\lambda$ and $K=1$ and also

$$
\frac{\left.\left|D X_{t}\right| E_{x}^{s}\right|_{*}}{\left.\left|D X_{t}\right| E_{x}^{X}\right|_{*}}=\left.\left|D X_{t}\right| E_{x}^{s}\right|_{*} \quad \text { and } \quad \frac{\left.\left|D X_{t}\right| E_{x}^{X}\right|_{*}}{\left.\left|D X_{-t}\right| E_{X_{t} x}^{u}\right|^{-1}}=\left|D X_{-t}\right| E_{X_{t} x}^{u} \mid
$$

are both bounded by $e^{-\lambda t}$ for all $t>0$. This shows that $|\cdot|_{*}$ is still adapted to the uniform hyperbolicity; and also adapted to the partially hyperbolic splitting $E^{s} \oplus\left(E^{X} \oplus E^{u}\right)$.

4.2.1. The norm is adapted to singular-hyperbolicity. With this norm, given by the inner product $(\cdot, \cdot)_{*}$, it is now easy to calculate

$$
\begin{aligned}
\left|\left(\wedge^{2} D X_{-t}\right)(X(x) \wedge v)\right|_{*}^{2} & =\left|D X_{-t} X(x)\right|_{*}^{2}\left|D X_{-t} v\right|_{*}^{2}-\left(D X_{-t} X(x), D X_{-t} v\right)_{*}^{2} \\
& \leq e^{-2 \lambda t}|v|_{*}^{2}=e^{-2 \lambda t}|v|_{*}^{2}|X(x)|_{*}^{2}=e^{-2 \lambda t}|X(x) \wedge v|_{*}^{2}
\end{aligned}
$$


for all $x \in \Gamma, t>0$ and all $u \in E_{x}^{u}$, since $E^{X}$ and $E^{u}$ are everywhere orthogonal by construction.

For any pair $u, v \in E_{x}^{u}$ and given fixed $t>0$, we can write $u \wedge v=u \wedge \tilde{v}$ so that $D X_{-t} \tilde{v}$ is orthogonal ${ }^{3}$ to $D X_{-t} u$. Thus

$$
\begin{aligned}
\left|\left(\wedge^{2} D X_{-t}\right)(u \wedge v)\right|_{*}^{2} & =\left|\left(\wedge^{2} D X_{-t}\right)(u \wedge \tilde{v})\right|_{*}^{2}=\left|D X_{-t} u\right|_{*}^{2}\left|D X_{-t} \tilde{v}\right|_{*}^{2} \\
& \leq e^{-4 \lambda t}|u|_{*}^{2}|\tilde{v}|_{*}^{2}=e^{-4 \lambda t}|u \wedge \tilde{v}|_{*}^{2}=e^{-4 \lambda t}|u \wedge v|_{*}^{2} .
\end{aligned}
$$

Hence, the norm of each elementary bivector, obtained from an orthonormal basis of $\wedge^{2}\left(E^{X} \oplus E^{c}\right)$ generated by vectors on $E^{X} \cup E^{c}$, is uniformly expanded by $\wedge^{2} D X_{t}$ at a rate of at least $\lambda$ with the norm $|\cdot|_{*}$. From Subsection 3.2.2, Lemma 3.4 and Remark 3.3, since we may apply the above inequalities to vectors from a singular basis for $\left.D X_{-t}\right|_{E^{X} \oplus E^{u}}$, this is enough to conclude that $\left\|\left.\wedge^{2} D X_{-t}\right|_{E^{X} \oplus E^{u}}\right\| \leq e^{-\lambda t}$ for each $t>0$.

Then this is a sectional-adapted norm to the splitting $E^{s} \oplus\left(E^{X} \oplus E^{u}\right)$.

The proof of Theorem B is complete.

4.3. The open class of sectional-hyperbolic sets. Here provide a proof of Theorem C.

Let $\Gamma$ be a sectional-hyperbolic set for a $C^{1}$ vector field whose splitting $T_{\Gamma} M=E \oplus$ $E^{c} \oplus E^{u}$ is dominated and such that: $E$ is uniformly contracted; $E^{c}$ two-dimensional and area expanding; $E^{u}$ is uniformly expanded and $E^{c} \oplus E^{u}$ is strongly partially hyperbolic. So there are $K>0$ and $\eta>\lambda>0$ such that for all $t>0$

(1) $\left\|\left.D X_{t}\right|_{E}\right\| \leq K e^{-\lambda t}$;

(2) $\left\|\left.D X_{t}\right|_{E}\right\| \leq K e^{-\lambda t} \mathrm{~m}\left(\left.D X_{t}\right|_{E^{c}}\right)$;

(3) $\left\|\left.D X_{t}\right|_{E^{c}}\right\| \leq K e^{\lambda t}$ and $\mathrm{m}\left(\left.D X_{t}\right|_{E^{u}}\right) \geq K e^{\eta t}$;

(4) $\left\|\wedge^{2} D X_{-t} \cdot\left(v_{1} \wedge v_{2}\right)\right\| \leq\left\|v_{1} \wedge v_{2}\right\| K e^{-\lambda t}$ for any $v_{1}, v_{2} \in E^{c}$.

Proof of Theorem C. Analogously to the proof of Theorem A, we introduce consecutively

- a skew-product flow $Z_{t}$ on $M \times \mathbb{S}^{1}$ admitting an invariant subset $\widetilde{\Gamma}=\Gamma \times\{S\}$ with a singular and sectional-hyperbolic splitting $E^{s} \oplus F_{0} \oplus E^{c} \oplus E^{u}$ of $T_{\widetilde{\Gamma}}\left(M \times \mathbb{S}^{1}\right)$ satisfying Remark 4.1, with $F_{0}$ in the place of $F$ and the same choice of inner product as in Subsection 4.1; together with

- a direct product flow $W_{t}=Z_{t} \times \bar{Y}_{t}:\left(M \times \mathbb{S}^{1}\right) \times \mathbb{S}^{1} \circlearrowleft$, where the vector field $\bar{Y}$ given by $f_{1}: \mathbb{S}^{1} \rightarrow \mathbb{R}$ is a North-South vector field as in the beginning of Subsection 4.1 and we choose $\alpha_{1}=f^{\prime}(1 / 2) \in(\lambda, \eta)$, where $1 / 2=N$ is the coordinate of the expanding fixed source at the north pole.

In this way, we get $D Y_{t} \mid T_{N} \mathbb{S}^{1}=e^{\alpha_{1} t}$ and $\widehat{\Gamma}=\widetilde{\Gamma} \times\{N\}=\Gamma \times\{S\} \times\{N\}$ admits a partially hyperbolic splitting $T_{\widehat{\Gamma}}=E^{s} \oplus F_{0} \oplus E^{c} \oplus F \oplus E^{u}$ where, using the inner product introduced in Subsection 4.1 making $F_{0} \oplus F$ orthogonal to $E^{s} \oplus E^{c} \oplus E^{u}$, we get

(1) $F_{0} \oplus E^{c}$ is a sectional- and singular-hyperbolic splitting (since $\operatorname{dim} E^{c}=2$ ); and

(2) $E^{c} \oplus F$ and $F \oplus E^{u}$ are both strongly partially hyperbolic splittings.

\footnotetext{
${ }^{3}$ Set $\tilde{v}=v-h u$ so that $h \cdot D X_{-t} u$ is the orthogonal projection of $D X_{-t} v$ along $D X_{-t} u$.
} 
The first item is a consequence of the arguments in the proof of Theorem A applied to the action of $Z_{t}$ over the subbundle $F_{0} \oplus E^{c}$; see Lemma 4.4 and the arguments following its proof.

The second item above is a consequence of the following estimates for a.e. point with respect to each $W_{t}$-invariant probability measure

$$
\begin{aligned}
& \lim _{t \rightarrow+\infty} \frac{1}{t} \log \left(\left\|\left.D W_{t}\right|_{E_{x}^{c}}\right\| \cdot\left\|\left.D W_{-t}\right|_{F_{W_{t} x}}\right\|\right) \leq \lambda-\alpha_{1}<0 \quad \text { and } \\
& \lim _{t \rightarrow+\infty} \frac{1}{t} \log \left(\left\|\left.D W_{t}\right|_{F_{x}}\right\| \cdot\left\|\left.D W_{-t}\right|_{E_{X_{t} x}^{u}}\right\|\right) \leq \alpha_{1}-\eta<0
\end{aligned}
$$

since $\left.D W_{t}\right|_{F_{x}}=D Y_{y} \mid T_{N} \mathbb{S}^{1}=e^{\alpha_{1} t}=\mathrm{m}\left(\left.D W_{t}\right|_{F_{x}}\right)$; coupled with [18, Theorem 1.9] providing domination of the splittings $E^{c} \oplus F$ and $F \oplus E^{u}$. More precisely, we set first $f_{t}(x)=\log \left(\left\|\left.D W_{t}\right|_{E_{x}^{c}}\right\| \cdot\left\|\left(\left.D W_{-t}\right|_{F_{W_{t}}}\right)^{-1}\right\|\right)$ and then again $f_{t}(x)=\log \left(\left\|\left.D W_{t}\right|_{F_{x}}\right\|\right.$. $\left.\left\|\left.D W_{-t}\right|_{E_{X_{t} x}^{u}} ^{u}\right\|\right)$ in the statement of the following result.

Lemma 4.9. [5, Corollary 4.2] Let $\left\{t \mapsto f_{t}: S \rightarrow \mathbb{R}\right\}_{t \in \mathbb{R}}$ be a continuous family of continuous functions on the $X_{t}$-invariant compact subset $S$, which is subadditive and suppose that there exists a constant $\lambda>0$ so that $\int\left(\lim _{t \rightarrow+\infty} \frac{1}{t} f_{t}(x)\right) d \eta \leq-\lambda$ for every $X_{t}$-invariant probability measure $\eta$. Then there exists a constant $K>0$ such that $\exp f_{t}(x) \leq K e^{-\lambda t}$ for every $x \in S$ and every $t>0$.

This provides constants $C_{1}, C_{2}>0$ so that, since $\left\|\left.D W_{t}\right|_{F}\right\|=e^{\alpha_{1} t}=\mathrm{m}\left(\left.D W_{t}\right|_{F}\right)$ independently of the norm, we obtain

$$
\begin{gathered}
\left\|\left.D W_{t}\right|_{E_{x}^{c}}\right\| \cdot\left\|\left.D W_{-t}\right|_{F_{W_{t} x}}\right\| \leq C_{1} e^{\left(\lambda-\alpha_{1}\right) t} \quad \text { and } \quad\left\|\left.D W_{t}\right|_{F_{x}}\right\| \cdot\left\|\left.D W_{-t}\right|_{E_{W_{t} x}^{u}}\right\| \leq C_{2} e^{\left(\alpha_{1}-\eta\right) t} \\
\text { and so }\left\|\left.D W_{t}\right|_{E_{x}^{c}}\right\| \leq C_{1} e^{\lambda t} \quad \text { and } \quad 1<C_{2} e^{-\eta t} \operatorname{m}\left(\left.D W_{t}\right|_{E_{x}^{u}}\right) .
\end{gathered}
$$

These relations, together with items (1-3), ensure that $E^{s} \oplus F_{0} \oplus E^{c} \oplus F \oplus E^{u}$ is a partially hyperbolic splitting: the splitting is dominated; $E^{s} \oplus F_{0}$ is uniformly contracted; and $F \oplus E^{u}$ uniformly expanded. Thus, we can assume without loss of generality that we have a Riemmanian metric $\|\cdot\|$ on $T_{\widehat{\Gamma}}\left(M \times\left(\mathbb{S}^{1}\right)^{2}\right)$ such that this splitting is orthogonal, $C_{1}=C_{2}=1$ in (4.6) together with

$$
\begin{aligned}
& \left\|D X_{t}\left|E^{s}\|=\| D W_{t}\right| E^{s}\right\| \leq e^{-\lambda t} \mathrm{~m}\left(D W_{t} \mid F_{0}\right) \\
& \left\|\left.D W_{t}\right|_{E_{x}^{c}}\right\| \leq e^{\lambda t}<e^{\alpha_{1} t}=\left\|\left.D W_{t}\right|_{F_{x}}\right\|<e^{\eta t} \leq\left\|\left.D W_{t}\right|_{E_{x}^{u}}\right\| ;
\end{aligned}
$$

and also $K=1$ on items (1-3), by using the result from [10].

In particular, note that the subbundle $F_{0} \oplus E^{c} \oplus F$ has a sectional-hyperbolic splitting. Indeed, we have that $F_{0}$ is uniformly contracted, $E^{c}$ is two-dimensional and area expanding, and $F$ is uniformly expanding; and thus $E^{c} \oplus F$ is sectionally-expanded. In fact, since the bundles are orthogonal we can argue as in Subsection 4.2.1: for any given fixed $t>0$, a unit vector $f_{1} \in F$ and a unit vector $v \in E^{c}$ we have, for any unit vector $\tilde{v} \in E^{c}$ such that 
$D X_{-t} \tilde{v}$ is othogonal to $D X_{-t} v$, the following bound

$$
\begin{aligned}
\left\|\left(\wedge^{2} D W_{-t}\right)\left(f_{1} \wedge v\right)\right\| & =\left\|D W_{-t} f_{1}\right\|^{2}\left\|D W_{-t} v\right\|^{2}-\left(D W_{-t} f_{1}, D W_{-t} v\right)^{2} \\
& =\left\|D W_{-t} f_{1}\right\|^{2}\left\|D W_{-t} v\right\|^{2} \\
& \leq e^{2\left(\lambda-\alpha_{1}\right) t}\left\|D W_{-t} \tilde{v}\right\|^{2}\left\|D W_{-t} v\right\|^{2}=e^{2\left(\lambda-\alpha_{1}\right) t}\left\|\left(\wedge^{2} D W_{-t}\right)(\tilde{v} \wedge v)\right\|^{2} \\
& \leq e^{2\left(\lambda-\alpha_{1}\right) t}\left\|\wedge^{2} D X_{-t} \mid \wedge^{2} E^{c}\right\|^{2} \leq e^{2\left(\lambda-\alpha_{1}\right) t} \cdot K^{2} e^{-2 \lambda t}=K^{2} e^{-2 \alpha_{1} t}
\end{aligned}
$$

where we have used the domination relations (4.6) in the following form

$$
1=\frac{\|\tilde{v}\|}{\left\|f_{1}\right\|}=\frac{\left\|D W_{t} \cdot D W_{-t} \tilde{v}\right\|}{\left\|D W_{t} \cdot D W_{-t} f_{1}\right\|}<e^{\left(\lambda-\alpha_{1}\right) t} \frac{\left\|D W_{-t} \tilde{v}\right\|}{\left\|D W_{-t} f_{1}\right\|}
$$

to pass from $f_{1}$ to $\tilde{v}$ together with item (4).

Hence, after Lemma 3.4, we have $\left\|\wedge^{2} D W_{-t} \mid \wedge^{2}\left(E^{c} \oplus F\right)\right\| \leq K e^{-\alpha_{1} t}, t>0$. Moreover, analogously, given $t>0$ and unit vectors $f_{0}, v$ on $F_{0}$ and $E^{c}$, respectively, choosing now a unit $\tilde{v} \in E_{x}^{c}$ such that $D W_{t} \tilde{v}$ is orthogonal to $D X_{t} v$, we obtain

$$
\begin{aligned}
\left\|\left(\wedge^{2} D W_{t}\right)\left(f_{0} \wedge v\right)\right\|^{2} & =\left\|D W_{t} f_{0}\right\|^{2} \cdot\left\|D W_{t} v\right\|^{2} \leq e^{-2 \lambda t}\left\|D W_{t} \tilde{v}\right\|^{2} \cdot\left\|D W_{t} v\right\|^{2} \\
& \leq e^{-2 \lambda t}\left\|\wedge^{2} D W_{t} \cdot \tilde{v} \wedge v\right\|^{2} \leq e^{-2 \lambda t}\left\|\left.\wedge^{2} D W_{t}\right|_{\wedge^{2} E^{c}}\right\|^{2} .
\end{aligned}
$$

This shows that the following is a dominated splitting of $G=\wedge^{2}\left(F_{0} \oplus E^{c} \oplus F\right)$ with respect to the action of $\wedge^{2} D W_{t} \mid G$ :

$$
G=\wedge^{2}\left(F_{0} \oplus E^{c}\right) \oplus\left(\left(F_{0} \oplus E^{c}\right) \wedge F\right)=G_{0} \oplus G_{1}
$$

where $G_{0}=\wedge^{2}\left(F_{0} \oplus E^{c}\right)$ and $G_{1}=\left(F_{0} \oplus E^{c}\right) \wedge F$ is the subbundle generated by the exterior products $u \wedge f$ for any $u \in F_{0} \oplus E^{c}$ and $f \in F$. In addition, $G_{0}$ also admits a partially hyperbolic splitting $F_{0} \wedge E^{c} \oplus \wedge^{2} E^{c}=\widetilde{F_{0}} \oplus \widetilde{E^{c}}$ just like in the proof of Theorem A

Thus, by [10], there exists an adapted norm $|\cdot|_{*}$ on $G$ for the action of $\wedge^{2} D W_{t} \mid G$ : this norm restricted to $G_{0}$ satisfies the assumptions of Lemma 4.6 with $m=3, E=F_{0}$ and $F=E^{c} ;$ and there exists $\xi>0$ so that for all $u, v, w \in F_{0} \oplus E^{c}$ and $t>0$

$$
\frac{\left|\wedge^{2} D W_{t} \cdot(u \wedge v)\right|_{*}}{\left|\wedge^{2} D W_{t} \cdot(w \wedge f)\right|_{*}}<e^{-\xi t} \frac{|u \wedge v|_{*}}{|w \wedge f|_{*}} .
$$

We observe that both $G_{0}=\wedge^{2}\left(F_{0} \oplus E^{c}\right) \simeq F_{0} \oplus E^{c}$ and $\wedge^{2}\left(E^{c} \oplus F\right) \simeq E^{c} \oplus F$ since the dimensions are the same. Hence, by Lemma 3.9, there are Riemannian norms $|\cdot|_{0}$ on $F_{0} \oplus E^{c}$ and $|\cdot|_{1}$ on $E^{c} \oplus F$ which induce $|\cdot|_{*}$ restricted to $G_{0}$ and $\wedge^{2}\left(E^{c} \oplus F\right)$, respectively. We write $\langle\cdot, \cdot\rangle_{i}$ for the innner products generating $|\cdot|_{i}, i=1,2$.

Applying Lemma 4.6 to $\left(G_{0},|\cdot|_{*}\right)$ and $\left(F_{0} \oplus E^{c},|\cdot|_{0}\right)$ we obtain (3.1) with $F_{0}$ in the place of $F$. Moreover, fixing $t>0$, if we define the Riemannian metric $\left|\alpha_{0} f_{0}+v+\alpha_{1} f\right|^{2}=$ $\beta^{2}\left|\alpha_{0} f_{0}+v\right|_{0}^{2}+\left|\alpha_{1} f\right|_{1}^{2}$ on $F_{0} \oplus E^{c} \oplus F$ for any given unit vector $v \in E^{c}$ and $\alpha_{0}, \alpha_{1} \in \mathbb{R}$ and some $\beta>0$, we get

$$
\frac{\left|D W_{t} v\right|}{\left|D W_{t} f\right|}=\beta \frac{\left|D W_{t} v\right|_{0}}{\left|D W_{t} f\right|_{1}}=\beta \frac{\left|\wedge^{2} D W_{t} \cdot(v \wedge \tilde{v})\right|_{*}}{\left|\wedge^{2} D W_{t} \cdot(f \wedge u)\right|_{*}} \leq e^{-\xi t} \cdot \beta \frac{|v \wedge \tilde{v}|_{*}}{|f \wedge u|_{*}}
$$


where $\tilde{v} \in E^{c}$ and $u \in E^{c} \oplus F_{1}$ are such that $\left|D W_{t} \tilde{v}\right|_{0}=1$ and $\left\langle D W_{t} \tilde{v}, D W_{t} v\right\rangle_{0}=0$ and $\left\langle D W_{t} u, D W_{t} f\right\rangle_{1}=0$. Note that the introduction of $\beta$ does not change any of the relations obtained for $|\cdot|_{0}$.

Now we argue similarly as in the proof of Lemma 4.6: we choose $\beta>0$ so that

$$
\beta \cdot \sup _{x \in \widehat{\Gamma}, 0<t \leq 1}\left\{\frac{\left|v \wedge D X_{-t} \tilde{v}\right|_{*}}{\left|f \wedge D X_{-t} u\right|_{*}}: v \in E_{x}^{c} ; \tilde{v}, u \in E_{X_{t} x}^{c}\right\} \leq 1 .
$$

This immediately provides the following for $0<t \leq 1$

$$
\left|D X_{t}\right| E^{c}|=| D W_{t}\left|E^{c}\right| \leq e^{-\xi t} \mathrm{~m}\left(D W_{t} \mid F\right)=e^{-\xi t} e^{\mu_{1} t} .
$$

For general $t>0$ we again apply Lemma 4.3 with $a=1$.

Now we check that the Riemannian metric defined by

$$
\left\|w+\alpha_{0} f_{0}+v+\alpha_{1} f+u\right\|_{*}^{2}=\|w\|^{2}+\left|\alpha_{0} f_{0}+v+\alpha_{1} f\right|^{2}+\|u\|^{2}
$$

for $w \in E_{x}^{s}, v \in E_{x}^{c}, u \in E_{x}^{u}, \alpha_{0} \cdot \alpha_{1} \in \mathbb{R}$ and $x \in \widehat{\Gamma}$ is a sectional-adapted metric when restricted to $E^{s} \oplus E^{c} \oplus E^{u}$.

We just have the use the previous estimates: we already have uniformly adapted contraction along $E^{s}$ and expansion along $E^{u}$ from items (1) and (3). For the domination relations, for $t>0$ we estimate ${ }^{4}$ using (4.6) and (4.7) and $|\cdot|=|\cdot|_{0}$ norm on $F_{0} \oplus E^{c}$

$$
\begin{aligned}
& \frac{\left\|D X_{t} \mid E^{s}\right\|_{*}}{\mathrm{~m}_{*}\left(D X_{t} \mid E^{c}\right)}=\frac{\left\|D X_{t} \mid E^{s}\right\|}{\left\|D W_{t} \mid F_{0}\right\|} \cdot \frac{\left\|D W_{t} \mid F_{0}\right\|}{\mathrm{m}_{*}\left(D W_{t} \mid E^{c}\right)}=e^{-\lambda t} \cdot \frac{\left|D W_{t}\right| F_{0} \mid}{\mathrm{m}\left(D W_{t} \mid E^{c}\right)} \leq e^{-\lambda t} e^{-\sigma t} ; \\
& \frac{\left\|D X_{t} \mid E^{c}\right\|_{*}}{\mathrm{~m}_{*}\left(D X_{t} \mid E^{u}\right)}=\frac{\left|D W_{t}\right| E^{c} \mid}{e^{\alpha_{1} t}} \cdot \frac{e^{\alpha_{1} t}}{\mathrm{~m}\left(D W_{t} \mid E^{u}\right)} \leq e^{-\xi t} \cdot \frac{\left\|D W_{t} \mid F\right\|}{\mathrm{m}\left(D W_{t} \mid E^{u}\right)} \leq e^{-\xi t} e^{-\eta t} ;
\end{aligned}
$$

where we have used $\left\|D W_{t}\left|F_{0} \|=\right| D W_{t}\left|F_{0}\right|\right.$ since $\operatorname{dim} F_{0}=1$ and analogously for $F$. For the sectional expansion, we already have for $t>0$

$$
\left\|\wedge^{2} D X_{t}\left|\wedge^{2} E^{c} \|_{*}=\right| \wedge^{2} D W_{t}\left|\wedge^{2} E^{c}\right|=\left.\left|\wedge^{2} D W_{t}\right| \wedge^{2} E^{c}\right|_{0} \geq e^{\sigma t}\right.
$$

for some $\sigma>0$. Since $E^{c}, F$ and $E^{u}$ are orthogonal for the metric inducing $\|\cdot\|_{*}$, for any given $v \in E^{c}, u \in E^{u}$ of unit norm, and $\tilde{v} \in E^{c}$ so that $\left\|D X_{-t} \tilde{v}\right\|_{*}=1$ and $\left\langle D X_{-t} \tilde{v}, D X_{-t} v\right\rangle_{*}=0$, we obtain using the domination

$$
\begin{aligned}
\left\|\wedge^{2} D X_{-t} \cdot(v \wedge u)\right\|_{*} & =\left\|D X_{-t} v\right\|_{*}\left\|D X_{-t} u\right\|_{*} \leq e^{-(\xi+\eta) t}\left\|D X_{-t} v\right\|_{*}\left\|D X_{-t} \tilde{v}\right\|_{*} \\
& =e^{-(\xi+\eta) t}\left\|\wedge^{2} D X_{-t} \cdot(v \wedge \tilde{v})\right\|_{*} \leq e^{-(\xi+\eta+\sigma) t}\|v \wedge \tilde{v}\|_{*} .
\end{aligned}
$$

Finally, for $u, v \in E^{u}$ we estimate just like in (4.4) to obtain

$$
\left\|\left(\wedge^{2} D X_{-t}\right)(u \wedge v)\right\|_{*}=\left\|\left(\wedge^{2} D X_{-t}\right)(u \wedge \tilde{v})\right\|_{*} \leq e^{-2 \eta t}\|u \wedge v\|_{*}
$$

where $u \wedge v=u \wedge \tilde{v}$ so that $D X_{-t} \tilde{v}$ is orthogonal to $D X_{-t} u$. This coupled with Lemma 3.4 is enough to conclude that $\left\|\wedge^{2} D X_{-t} \mid \wedge^{2}\left(E^{c} \oplus E^{u}\right)\right\|_{*}<e^{-2 \min \{\eta, \sigma\} t}$ for each $t>0$.

This completes the proof of Theorem $C$.

\footnotetext{
${ }^{4}$ We write $m_{*}$ for the conorm associated to the norm $\|\cdot\|_{*}$.
} 


\section{REFERENCES}

[1] V. Araujo. Sinks and sources for C1 dynamics whose Lyapunov exponents have constant sign. Kyoto Journal of Mathematics (to appear), pages 1-36, 2020.

[2] V. Araujo and M. J. Pacifico. Three-dimensional flows, volume 53 of Ergebnisse der Mathematik und ihrer Grenzgebiete. 3. Folge. A Series of Modern Surveys in Mathematics [Results in Mathematics and Related Areas. 3rd Series. A Series of Modern Surveys in Mathematics]. Springer, Heidelberg, 2010. With a foreword by Marcelo Viana.

[3] V. Araujo and L. Salgado. Infinitesimal Lyapunov functions for singular flows. Mathematische Zeitschrift, 275(3-4):863-897, 2013.

[4] V. Araujo and L. Salgado. Dominated splitting for exterior powers and singular-hyperbolicity. Journal of Differential Equations, 259(8):3874 - 3893, 2015.

[5] A. Arbieto and L. Salgado. On critical orbits and sectional-hyperbolicity of the nonwandering set for flows. Journal of Differential Equations, 250:2927-2939, 2010.

[6] L. Arnold. Random dynamical systems. Springer-Verlag, Berlin, 1998.

[7] C. Bonatti, L. J. Díaz, and M. Viana. Dynamics beyond uniform hyperbolicity, volume 102 of Encyclopaedia of Mathematical Sciences. Springer-Verlag, Berlin, 2005. A global geometric and probabilistic perspective, Mathematical Physics, III.

[8] R. Bowen. Equilibrium states and the ergodic theory of Anosov diffeomorphisms, volume 470 of Lect. Notes in Math. Springer Verlag, 1975.

[9] R. Bowen and D. Ruelle. The ergodic theory of Axiom A flows. Invent. Math., 29:181-202, 1975.

[10] N. Gourmelon. Adapted metrics for dominated splittings. Ergodic Theory Dynam. Systems, 27(6):1839-1849, 2007.

[11] M. Hirsch, C. Pugh, and M. Shub. Invariant manifolds, volume 583 of Lect. Notes in Math. Springer Verlag, New York, 1977.

[12] A. Katok and B. Hasselblatt. Introduction to the modern theory of dynamical systems, volume 54 of Encyclopeadia Appl. Math. Cambridge University Press, Cambridge, 1995.

[13] J. Lewowicz. Lyapunov functions and topological stability. J. Differential Equations, 38(2):192-209, 1980.

[14] R. Metzger and C. Morales. Sectional-hyperbolic systems. Ergodic Theory and Dynamical System, 28:1587-1597, 2008.

[15] C. A. Morales, M. J. Pacifico, and E. R. Pujals. singular-hyperbolic systems. Proc. Amer. Math. Soc., 127(11):3393-3401, 1999.

[16] C. A. Morales, M. J. Pacifico, and E. R. Pujals. Robust transitive singular sets for 3-flows are partially hyperbolic attractors or repellers. Ann. of Math. (2), 160(2):375-432, 2004.

[17] C. Robinson. An introduction to dynamical systems: continuous and discrete. Pearson Prentice Hall, Upper Saddle River, NJ, 2004.

[18] L. Salgado. singular-hyperbolicity and sectional Lyapunov exponents of various orders. Proc. Amer. Math. Soc., 147(2):735-749, 2019.

[19] L. Salgado and V. Coelho. Adapted metrics for codimension one singular-hyperbolic flows. In M. J. Pacifico and P. Guarino, editors, New Trends in One-Dimensional Dynamics, pages 249-269. Springer International Publishing, Cham, 2019.

[20] M. Shub. Global stability of dynamical systems. Springer Verlag, 1987.

[21] W. Tucker. The Lorenz attractor exists. C. R. Acad. Sci. Paris, 328, Série I:1197-1202, 1999.

[22] D. V. Turaev and L. P. Shil'nikov. An example of a wild strange attractor. Mat. Sb., 189(2):137-160, 1998.

[23] M. Viana. What's new on Lorenz strange attractor. Mathematical Intelligencer, 22(3):6-19, 2000.

[24] S. Winitzki. Linear Algebra: Via Exterior Products. Lulu.com, Raleigh, N.C., USA, 1.2 edition, 2012. 
[25] M. P. Wojtkowski. Monotonicity, J-algebra of Potapov and Lyapunov exponents. In Smooth ergodic theory and its applications (Seattle, WA, 1999), volume 69 of Proc. Sympos. Pure Math., pages 499521. Amer. Math. Soc., Providence, RI, 2001.

(V.A.) Universidade Federal da Bahia, Instituto de Matemática e Estatística, Av. Adhemar de BARros, S/N , Ondina, 40170-110 - SAlvador-BA-Brazil

Email address: vitor.d.araujo@ufba.br, vitor.araujo.im.ufba@gmail.com

(V.C.) Universidade Federal do Oeste da Bahia, Centro Multidisciplinar de Bom Jesus da Lapa, Av. Manoel Novais, 1064, Centro, 47600-000 - Bom Jesus da Lapa-BA-Brazil

Email address: viniciuscs@ufob.edu.br

(L.S.) Universidade Federal do Rio de Janeiro, Instituto de Matemática, Avenida Athos da Silveira Ramos 149 Cidade Universitária, P.O. Box 68530, 21941-909 Rio de Janeiro-RJBRAZIL

Email address: lsalgado@im.ufjr.br, lucianasalgado@ufrj.br 